\title{
Effect of Polyphenol-Rich Foods, Juices, and Concentrates on Recovery from Exercise Induced Muscle Damage: A Systematic Review and Meta-Analysis
}

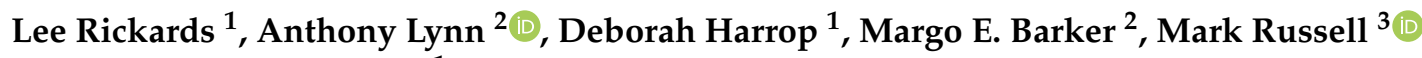 \\ and Mayur K. Ranchordas ${ }^{1, *}$ \\ 1 Academy of Sport \& Physical Activity, Sheffield Hallam University, Sheffield S10 2BP, UK; \\ lr7134@exchange.shu.ac.uk (L.R.); itsdh1@exchange.shu.ac.uk (D.H.) \\ 2 Department of Service Sector, Management Business School, Sheffield Hallam University, \\ Sheffield S1 1WP, UK; T.lynn@shu.ac.uk (A.L.); sbsmb@exchange.shu.ac.uk (M.E.B.) \\ 3 School of Social and Health Sciences, Leeds Trinity University, Leeds LS18 5HD, UK; \\ m.russell@leedstrinity.ac.uk \\ * Correspondence: m.ranchordas@shu.ac.uk; Tel.: +44-11-4225-5678
}

\section{check for} updates

Citation: Rickards, L.; Lynn, A.; Harrop, D.; Baker, M.E.; Russell, M.; Ranchordas, M.K. Effect of Polyphenol-Rich Foods, Juices, and Concentrates on Recovery from Exercise Induced Muscle Damage: A Systematic Review and Meta-Analysis. Nutrients 2021, 13, 2988. https://doi.org/10.3390/ nu13092988

Academic Editor: Daniel Hackett

Received: 12 July 2021

Accepted: 25 August 2021

Published: 27 August 2021

Publisher's Note: MDPI stays neutral with regard to jurisdictional claims in published maps and institutional affiliations.

Copyright: (c) 2021 by the authors. Licensee MDPI, Basel, Switzerland. This article is an open access article distributed under the terms and conditions of the Creative Commons Attribution (CC BY) license (https:// creativecommons.org/licenses/by/ $4.0 /)$.
Abstract: Objectives. To determine the effects of consuming polyphenol-rich foods, juices and concentrates on recovery from exercise-induced muscle damage (EIMD). Method. Eligibility criteria. Randomised and quasi-randomised placebo-controlled trials with a parallel or cross-over design evaluating the effects of consuming polyphenol-rich foods, juices and concentrates on recovery from EIMD in humans. Eligible studies included at least one of the primary outcome measures: maximal isometric voluntary contraction; MIVC, delayed onset muscle soreness; DOMS, or countermovement jump; CMJ. Information sources. AMED, Cochrane Central Register of Controlled Trials, International Clinical Trials Registry Platform, PUBMED, SCOPUS (Elsevier), SPORTDiscus (EBSCO), and the UK Clinical Trials Gateway were searched from inception to September 2020. Risk of bias and quality of evidence. Risk of bias was assessed using Cochrane Risk of Bias 2 tool. Quality of the evidence was assessed using the Grading of Recommendations, Assessment, Development and Evaluation framework. Synthesis of results. Random effects models were used to determine the effect of polyphenol supplementation on recovery from EIMD. Data are presented as standardised mean differences (SMD) with $95 \%$ confidence intervals (CI). Results. Included studies. Twenty-five studies were included; 15 had a parallel, and 10 had a cross-over design. A total of 527 participants (male: $n=425$; female: $n=102$ ) were included in the meta-analysis. Synthesis of results. Consumption of polyphenol-rich foods, juices and concentrates accelerated recovery of MIVC immediately post-exercise (SMD $=0.23,95 \% \mathrm{CI} 0.04,0.42 ; p=0.02 ;$ low-quality evidence), $24 \mathrm{~h}$ (SMD $=0.39,95 \%$ CI 0.15, 0.62; $p=0.001$; low-quality evidence), $48 \mathrm{~h}$ (SMD $=0.48,95 \%$ CI 0.28, 0.67; $p<0.001$; moderate-quality evidence), $72 \mathrm{~h}(\mathrm{SMD}=0.29,95 \% \mathrm{CI} 0.11,0.46 ; p=0.001$; low-quality evidence) and $96 \mathrm{~h}$ post-exercise ( $\mathrm{SMD}=0.50,95 \% \mathrm{CI} 0.16,0.83 ; p=0.004$; very low-quality evidence). DOMS was reduced at $24 \mathrm{~h}(\mathrm{SMD}=-0.29,95 \% \mathrm{CI}-0.47,-0.11 ; p=0.002$; low-quality evidence), $48 \mathrm{~h}$ (SMD $=-0.28,95 \% \mathrm{CI}-0.46,-0.09 ; p=0.003$; low-quality evidence) and $72 \mathrm{~h}$ post-exercise (SMD $=-0.46,95 \% \mathrm{CI}-0.69,-0.24 ; p<0.001$; very low-quality evidence). CMJ height was greater immediately post-exercise (SMD $=0.27,95 \%$ CI $0.01,0.53 ; p=0.04$; low-quality evidence), at $24 \mathrm{~h}$ $(\mathrm{SMD}=0.47,95 \%$ CI $0.11,0.83 ; p=0.01$; very low-quality evidence), $48 \mathrm{~h}$ (SMD $=0.58,95 \%$ CI 0.24 , $0.91 ; p<0.001$; very low-quality evidence) and $72 \mathrm{~h}$ post-exercise (SMD $=0.57,95 \% \mathrm{CI} 0.03,1.10$; $p=0.04$; very low-quality evidence). Polyphenol supplementation did not alter creatine kinase, c-reactive protein, and interleukin -6 at any time points. At $72 \mathrm{~h}$ post-exercise, protein carbonyls $(\mathrm{SMD}=-0.64,95 \% \mathrm{CI}-1.14,-0.14 ; p=0.01)$ were reduced. Discussion. Limitations of evidence. Risk of bias was high for 10 studies and moderate for 15. Sensitivity analyses excluding the high risk of bias studies reduced the SMDs for MIVC and DOMS, and for CMJ effects at 24 and $48 \mathrm{~h}$ were no longer statistically significant. Interpretation. Consuming polyphenol-rich foods, juices and concentrates accelerated recovery of muscle function while reducing muscle soreness in humans. Maximal benefit occurred $48-72 \mathrm{~h}$ post-exercise, however, the certainty of the evidence was moderate 
to very low. Supplementation could be useful when there is limited time between competitive events and impaired recovery could negatively impact performance.

Keywords: polyphenols; muscle damage; recovery; supplementation; exercise

\section{Introduction}

Exercise of a high intensity and/or duration, especially with an eccentric component, can induce muscle damage [1,2]. Exercise-induced muscle damage (EIMD) is characterised by impaired force production, increased muscle soreness and reduced range of motion $[1,3]$. These symptoms can impair subsequent performance. Therefore, sport nutrition strategies often aim to attenuate EIMD and accelerate recovery to enhance readiness to play or train. This is especially important when recovery time is reduced, such as during tournaments, multi-day events or periods of competition congestion.

The exact mechanisms underpinning EIMD have yet to be fully elucidated, but a two phase process has been proposed [4]. Firstly, mechanical damage to sarcomeres may cause overstretching of some filaments (sarcomere 'popping') [5] resulting in Z-band streaming and a loss of force production [6]. Secondly, an acute inflammatory response and disruption of redox balance may further damage the muscle [7]. Because inflammation and disruption of redox balance are implicated in the secondary phase of muscle damage, a growing number of studies have investigated whether foods with antioxidant and antiinflammatory properties accelerate recovery.

Polyphenols are secondary plant metabolites attributed with antioxidant and antiinflammatory properties [8]. Connolly et al. [9] were the first to demonstrate that a polyphenol-rich tart cherry/apple juice blend could accelerate recovery from eccentric elbow flexion. Accordingly, studies have subsequently investigated the efficacy of a large number of other foods and extracts rich in polyphenols including pomegranate [10], bilberry [11], blueberry [12], beetroot [13] and cocoa [14]. The results of these studies have been inconsistent, potentially due to methodological variation, including differences in exercise protocols, intervention periods, outcome markers, dosages, and the polyphenol content and bioactive constituents of the supplements consumed (e.g., beetroot is rich in nitrate, betalains and polyphenols) $[15,16]$.

To our knowledge, only one meta-analysis has synthesised the evidence on fruitderived polyphenols and recovery from EIMD [17]. Supplementation elicited a faster recovery of maximal isometric voluntary contraction (MIVC), increased antioxidant capacity and reduced markers of muscle damage, inflammation, and oxidative stress. However, this meta-analysis only reviewed evidence at 24 and $48 \mathrm{~h}$ post exercise, even though many of the included studies measured outcomes beyond this point and the symptoms of EIMD often take longer than $48 \mathrm{~h}$ to resolve. It is also difficult to interpret the results of Doma et al. [17] for blood markers of recovery because they pooled results from different biochemical assays that are not directly comparable.

Doma et al. [17] investigated the effects of polyphenol-rich food and isolated extracts in their meta-analysis. However, we recently found that in elite football, practitioners preferred to recommend polyphenol-rich fruits and vegetables rather than supplements of isolated compounds or extracts (manuscript in preparation). As different foods have distinct polyphenolic profiles it is important to explore their individual efficacy. Doma et al. [17] did not address this issue in their review nor did they evaluate the effects of restricting polyphenols in the background diet which could also confound the results of studies. Given these limitations, the main aim of this meta-analysis was to assess the effects of polyphenol-rich fruits and vegetables on recovery of EIMD up to $96 \mathrm{~h}$ post-exercise. The primary outcome markers chosen to assess recovery in this meta-analysis were MIVC, countermovement jump (CMJ) and delayed onset muscle soreness (DOMS). MIVC and $\mathrm{CMJ}$ both measure muscle force which has been argued to be the most appropriate marker 
of muscle damage [18]. Whereas DOMS is a commonly used marker of exercise-induced muscle pain [18]. The secondary outcomes assessed were blood markers of muscle damage (creatine kinase), inflammation (c-reactive protein, interleukin-6), and oxidative stress (protein carbonyls). These were selected because they have been commonly used in the literature to measure the extent of muscle damage and/or recovery from the secondary phase of EIMD [3].

Additional aims of this review were to explore differences in the effects of individual polyphenol-rich fruit/vegetable products on recovery from EIMD and to investigate whether restricting polyphenols in the background diet influences the efficacy of supplementation.

\section{Materials and Methods}

The protocol for this review was registered on the International Prospective Register of Systematic Reviews (PROSPERO) (CRD42018097123; 4 June 2018) and undertaken according to the guidelines of the Preferred Reporting Items for Systematic Reviews and MetaAnalysis (PRIMSA) [19] and the Cochrane handbook [20]. The original protocol specified this review was limited to studies with a parallel design because in cross-over designs susceptibility to muscle damage might be reduced on second exposure to eccentric exercise, even in the contralateral limb, a phenomenon known as the repeated bout effect [21]. Acknowledging that many studies in this area used a cross-over design, we have presented results for each study design independently and then overall as a combined result. Studies on isolated polyphenols/extracts were excluded because prior work conducted with sport nutrition practitioners indicated that $>70 \%$ preferred to recommend whole foods rather than extracts and isolated polyphenols (manuscript in preparation).

\subsection{Eligibility Criteria}

We included randomised and quasi-randomised controlled trials with a parallel or cross-over design published in English. Included studies compared a polyphenolrich food, juice or concentrate with a placebo on recovery from EIMD. Participants were aged $>16$ years, male or female, trained or untrained, and free from chronic disease. We included studies that: (1) used an exercise protocol designed to induce muscle damage; (2) investigated at least one of the following outcomes post-exercise: MIVC, DOMS or CMJ height. Studies were excluded if: (1) the polyphenol was not provided as a food, juice or concentrate; (2) polyphenol foods were combined with other supplements; (3) there was no control for practices that could have influenced recovery (e.g., simultaneous or additive use of compression garments, cold water immersion, other analgesic medication etc.).

\subsection{Search Strategy}

Studies that investigated the effects of polyphenols on recovery were identified by searching in the following databases from inception to September 2020: AMED, Cochrane Central Register of Controlled Trials, International Clinical Trials Registry Platform, PUBMED, SCOPUS (Elsevier), SPORTDiscus (EBSCO), and the UK Clinical Trials Gateway for ongoing, discontinued and completed studies. In addition, we performed citation chaining using identified studies to find other relevant publications. Details of our full search strategy is shown in online Supplementary File 1.

\subsection{Study Selection}

Eligibility of the studies was performed by two independent reviewers (LR and MKR) in a standardised manner. Titles, abstracts, and descriptors of the trials retrieved from the searches were independently screened. Studies that satisfied the inclusion criteria were selected and the full text reviewed. In the case of disagreements a third reviewer (AL) was consulted. 


\subsection{Data Extraction}

Two reviewers (LR and MKR) independently extracted data from the included studies using a pre-piloted data extraction form. Disagreements were resolved by discussion and consultation with a third reviewer (AL). When necessary, we contacted authors for additional information and data not reported in their manuscript. When data were not available in the manuscripts and authors did not respond to our contact requests, data were extracted from graphs in the published manuscript using Origin Pro $2020 b$ (Northampton, MA, USA, 2020), where possible. Information was extracted from each included study on: (1) characteristics of study participants (including age, sex, physical activity level); (2) type of intervention (including type, dosage, duration, and frequency of polyphenol supplementation); (3) means and standard deviations (SD) for each outcome measure (MIVC, DOMS, CMJ, creatine kinase; CK, C-reactive protein; CRP, interleukin-6; IL-6, and protein carbonyls; PC). Where standard errors were reported, we converted these to SDs using Review Manager [22].

\subsection{Risk of Bias in Individual Studies}

Included studies were independently assessed for risk of bias by two reviewers (AL and MKR). Studies with a parallel design were assessed using the Risk of Bias 2 tool (ROB 2) [23] and this was adapted for cross-over studies using guidelines provided by Higgins et al. [24]. Each study was assessed for quality across the following categories: (1) randomisation process; (2) deviations from intended interventions; (3) missing outcome data; (4) measurement of the outcome; (5) selection of the reported result. The overall assessment comprised of three ratings: low risk of bias, some concerns or high risk of bias. We resolved any disagreement by consulting a third reviewer (LR). Publication bias was investigated by visually inspecting funnel plots for asymmetry (see Supplementary File 2).

\subsection{Data Synthesis}

Meta-analyses were conducted using Review Manager [22]. We converted the data to SMDs for all primary and secondary outcomes because of differences in the units of measurement reported across studies. For studies with a parallel design, extracted means, SDs and number of participants were inputted into Review Manager to calculate the SMDs. The computed $95 \%$ CIs were used to calculate standard errors of the SMDs using the following equation [20]:

$$
\frac{\text { Upper } 95 \% \text { CI - Lower } 95 \% \text { CI }}{3.92}
$$

For studies with a cross-over design, the extracted means, SDs and number of participants were used to calculate the SD pooled, SMD, and standard error of the SMD using the following equations [20]:

$$
\begin{gathered}
S M D=\frac{M D}{S D_{\text {pooled }}} \\
S D_{\text {pooled }}=\sqrt{\frac{S D_{E}^{2}+S D_{C}^{2}}{2}} \\
S E(S M D)=\sqrt{\frac{1}{N}+\frac{S M D^{2}}{2 N}} \times \sqrt{2(1-\text { Corr })}
\end{gathered}
$$

When the raw data were available from cross-over trials we calculated the correlation between repeated measures. If no data were available we used a conservative correlation of 0.5 [20]. Some studies reported multiple measures of primary outcome markers (e.g., DOMS or MIVC at several anatomical sites $[10,25,26]$; or compared more than one treatment to a single placebo group $[25,27,28]$. To avoid these studies contributing more than one set of data (causing a unit of analysis error), we calculated combined means and SDs to produce a single SMD for each outcome marker at each time point per study [20,29]. Sensitivity 
analyses were conducted by removing the studies classified as high risk of bias to assess the robustness of treatment effects.

The polyphenol-rich foods included in this review differ in their content of polyphenols and other compounds that might promote recovery. Therefore, when two or more studies on an individual polyphenol-rich food were available, we conducted separate meta-analyses for the primary outcomes. To test whether polyphenol-rich foods were statistically different from each other we used the test for subgroup differences available in RevMan [22]. The efficacy of polyphenol supplementation to accelerate recovery may be influenced by the quantity of polyphenols in the background diet of participants. Therefore, we conducted subgroup analyses for our primary outcomes to compare studies that restricted the quantity of polyphenols in the background diet with those that did not.

SMDs were interpreted using the classifications of $0.2,0.5$ and 0.8 as small, moderate, and large effects, respectively [30]. Statistical significance was set at $p \leq 0.05$. To aid the interpretation of the SMDs of our primary outcome markers, effect sizes were converted into percentages. First the SMDs were converted into mean differences (MDs) by multiplying them by estimates of the SDs associated with the most frequently used unit of measurement (MFU) (MIVC: N, DOMS: $\mathrm{cm}$ and CMJ height: $\mathrm{cm}$ ) [31]. Second, these calculated MDs were converted into percentages:

$$
\frac{M D}{\text { Mean of MFU placebo group }} \times 100
$$

We assessed heterogeneity of data by visually inspecting forest plots, and conducting the $\mathrm{I}^{2}$ test and $\chi^{2}$ test. We considered an $\mathrm{I}^{2}$ value greater than $40 \%$ and/or a $\chi^{2} p$ value of less than 0.1 as evidence of substantial heterogeneity [20]. For a number of outcome markers there was substantial heterogeneity for one or more time points, therefore, we used the random effects model for all of the analyses. When applied to homogeneous studies, the random effects model produces the same estimate of effect as the fixed effect model [32].

\subsection{Quality Assessment}

Overall quality of evidence was assessed by two independent reviewers (AL and MKR) using the Grading of Recommendations, Assessment, Development and Evaluation framework (GRADE) [33]. Any disagreements were resolved by consulting a third reviewer (LR). Overall quality of evidence for each primary outcome was rated from high to very low. Quality was assessed against the following five factors: (1) study limitations; (2) imprecision; (3) inconsistency of results; (4) indirectness of evidence; (5) publication bias (see Supplementary File 3).

\section{Results}

\subsection{Study Selection}

A total of 25 studies were identified for inclusion in this review. The search of AMED, Cochrane Central Register of Controlled Trials, International Clinical Trials Registry, PUBMED, SCOPUS (Elsevier), SPORTDiscus (EBSCO), and UK Clinical Trials Gateway identified a total of 15,133 records and a further three studies were identified through citation chaining. Of these, 13,420 were screened after removing 1716 duplicates. After reviewing the abstracts 13,333 were removed because they did not meet our inclusion criteria. The full text of the remaining 87 studies were examined in more detail and 62 were removed (see flow diagram for reasons Figure 1). 


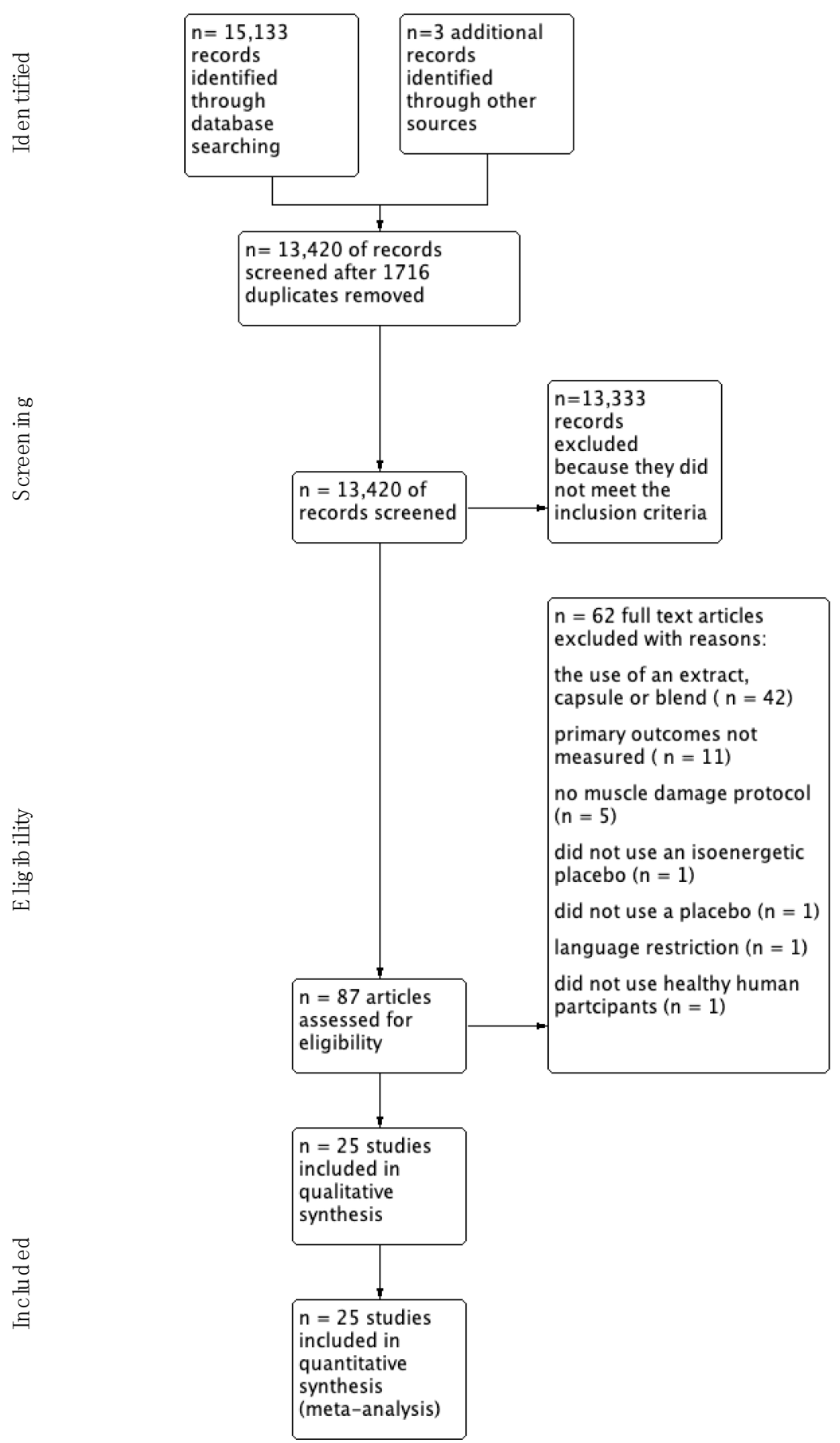

Figure 1. Study Flow Diagram.

\subsection{Study Characteristics}

\subsubsection{Methods}

Of the 25 studies included in the review, 15 had a parallel design and 10 employed a cross-over design (see Table 1). Participants were randomised to treatment groups in 20 studies, whereas, a quasi-randomisation protocol was used in one cross-over [34] and four parallel studies [27,35-37]. In 18 studies both investigators and participants were blinded to the treatment allocation $[10,13,14,25,27,28,34,35,37-46]$, in 4 studies investigators were aware of the treatment allocation $[11,26,47,48]$ and in three studies there was no/unclear information on blinding $[9,12,36]$. The duration of supplementation ranged from 1 to 15 days. 
Table 1. Characteristics of the studies included in the systematic review and meta-analysis.

\begin{tabular}{|c|c|c|c|c|c|c|}
\hline Authors & $\begin{array}{c}\text { Participant } \\
\text { Characteristics at } \\
\text { Baseline }\end{array}$ & $\begin{array}{l}\text { Study } \\
\text { Design }\end{array}$ & $\begin{array}{c}\text { Exercise } \\
\text { Intervention }\end{array}$ & Polyphenol Supplement & $\begin{array}{l}\text { Dosage and } \\
\text { Duration }\end{array}$ & $\begin{array}{l}\text { Outcome Variables and } \\
\text { Time of Measurement } \\
\text { (h) }\end{array}$ \\
\hline $\begin{array}{l}\text { Abbot } \\
\text { et al. } 2019 \\
{[38]}\end{array}$ & $\begin{array}{c}\text { Professional male } \\
\text { soccer players } \\
\text { Age } 19 \pm 1 \mathrm{y} \\
\text { Height } 1.8 \pm 0.6 \mathrm{~m} \\
\text { Mass } 77.3 \pm 6.4 \mathrm{~kg} \\
n=10\end{array}$ & Crossover & $\begin{array}{l}90 \text { min soccer } \\
\text { match }\end{array}$ & $\begin{array}{c}\text { Tart cherry juice } \\
\text { Polyphenol content not } \\
\text { stated }\end{array}$ & $\begin{array}{c}2 \times 30 \mathrm{~mL} \\
\text { concentrate for } \\
3 \text { days } \\
\text { (morning of } \\
\text { match until } 36 \\
\mathrm{~h} \\
\text { post-exercise) }\end{array}$ & $\begin{array}{c}\text { DOMS }(12,36,60) \\
\text { CMJ }(12,36,60)\end{array}$ \\
\hline $\begin{array}{l}\text { Bell et al. } \\
2015 \text { [39] }\end{array}$ & $\begin{array}{c}\text { Male healthy trained } \\
\text { cyclists } \\
\text { Age } 30 \pm 8 \text { years } \\
\text { Height } 181.1 \pm 6.7 \mathrm{~cm} \\
\text { Mass } 76.5 \pm 9.2 \mathrm{~kg} \\
\dot{V} \mathrm{O}_{2 \text { peak }} 61.6 \pm 10.4 \\
\mathrm{~mL} \cdot \mathrm{kg}^{-1} \cdot \mathrm{min}^{-1} \\
n=16\end{array}$ & Parallel & $\begin{array}{l}109 \text { min } \\
\text { stochastic } \\
\text { cycling }\end{array}$ & $\begin{array}{c}\text { Montmorency tart cherry } \\
\text { juice } \\
\text { Per } 1 \mathrm{~mL} \\
9.2 \mathrm{mg} \text { of anthocyanins } \\
\text { (HPLC) } \\
\text { Atlas Biosciences, Tuscon, } \\
\text { Arizona, USA }\end{array}$ & $\begin{array}{c}2 \times 30 \mathrm{~mL} \\
\text { concentrate for } \\
8 \text { days }(4 \text { days } \\
\text { pre-exercise, } \\
\text { on the day of, } \\
\text { and } 3 \text { days } \\
\text { post-exercise) }\end{array}$ & $\begin{array}{c}\text { MIVC (PE, 24, 48, 72) } \\
\text { DOMS (PE, 24, 48, 72) } \\
\text { CK (PE, 24, 48, 72) } \\
\text { CRP (PE, 24, 48, 72) } \\
\text { IL-6 (PE, 24, 48, 72) }\end{array}$ \\
\hline $\begin{array}{l}\text { Bell et al. } \\
2016 \text { [40] }\end{array}$ & $\begin{array}{c}\text { Male } \\
\text { semi-professional } \\
\text { soccer players } \\
\text { Age } 25 \pm 4 \text { years } \\
\text { Height } 180.8 \pm 7.4 \mathrm{~cm} \\
\text { Mass } 81.9 \pm 6.6 \mathrm{~kg} \\
\dot{V} \mathrm{O}_{2 \text { peak }} 54.9 \\
\mathrm{~mL} \cdot \mathrm{kg}^{-1} \cdot \mathrm{min}^{-1} \\
n=16\end{array}$ & Parallel & $\begin{array}{l}\text { Loughborough } \\
\text { intermittent } \\
\text { shuttle test }\end{array}$ & $\begin{array}{c}\text { Montmorency tart cherry } \\
\text { juice } \\
\text { Per } 1000 \mathrm{~mL} \\
73.5 \mathrm{mg} \\
\text { cyanidin-3-glucoside } \\
\text { (HPLC) } \\
\text { 178.8 mg of GAE (total } \\
\text { phenols) } \\
0.58 \text { trolox equivalent } \\
\text { (TEAC) } \\
\text { Based on previous work } \\
\text { from own laboratory (61) }\end{array}$ & $\begin{array}{c}2 \times 30 \mathrm{~mL} \\
\text { concentrate for } \\
8 \text { days }(4 \text { days } \\
\text { pre-exercise, } \\
\text { on the day of, } \\
\text { and } 3 \text { days } \\
\text { post-exercise) }\end{array}$ & $\begin{array}{l}\text { MIVC (PE, 24, 48, 72) } \\
\text { DOMS PE, 24, 48,72) } \\
\text { CMJ (PE, 24, 48,72) } \\
\text { CK (PE, 24, 48, 72) } \\
\text { CRP (PE, 24, 48, 72) } \\
\text { IL-6 (PE, 24, 48, 72) }\end{array}$ \\
\hline $\begin{array}{l}\text { Bowtell } \\
\text { et al. 2011 } \\
\text { [34] }\end{array}$ & $\begin{array}{c}\text { Male well-trained } \\
\text { participants } \\
\text { Age } 27.8 \pm 1.6 \text { years } \\
\text { Height } 1.76 \pm 0.03 \mathrm{~m} \\
\text { Mass } 81.3 \pm 4.3 \mathrm{~kg} \\
n=10\end{array}$ & Crossover & $\begin{array}{l}10 \times 10 \text { single } \\
\text { leg extension }\end{array}$ & $\begin{array}{c}\text { Montmorency tart cherry } \\
\text { juice } \\
275 \mathrm{mmol} \cdot \mathrm{L}^{-1} \text { trolox } \\
\text { equivalents (ORAC) } \\
\text { (Brunswick Laboratories, } \\
\text { Southborough, USA) } \\
\text { Per } 1 \mathrm{~mL} \\
9.117 \mathrm{mg} \text { of anthocyanins: } \\
\text { malvidin (4.696 mg) and } \\
\text { cyanidin ( } 3.346 \mathrm{mg} \text { ) } \\
\text { (HPLC) (Atlas, } \\
\text { Biosciences, Tucson, } \\
\text { Arizona, USA) }\end{array}$ & $\begin{array}{c}2 \times 30 \mathrm{~mL} \\
\text { concentrate for } \\
10 \text { days ( } 7 \\
\text { days } \\
\text { pre-exercise, } \\
\text { on the day of, } \\
\text { and } 2 \text { days } \\
\text { post-exercise) }\end{array}$ & $\begin{array}{c}\text { MIVC }(\mathrm{PE}, 24,48) \\
\text { CK }(24,48) \\
\text { CRP }(\mathrm{PE}, 24,48) \\
\text { PC (PE, 24, 48) }\end{array}$ \\
\hline $\begin{array}{l}\text { Brown, } \\
\text { Stevenson } \\
\quad \text { and } \\
\text { Howatson } \\
\text { (2019) [41] }\end{array}$ & $\begin{array}{c}\text { Female physically } \\
\text { active females } \\
\text { Age } 19 \pm 1 \text { years } \\
\text { Height } 167 \pm 6 \mathrm{~cm} \\
\text { Mass } 61.4 \pm 5.7 \mathrm{~kg} \\
\text { BMI } 22.1 \pm 1.9 \mathrm{~kg} \\
\mathrm{~m}^{-2} \\
n=20\end{array}$ & Parallel & $\begin{array}{l}15 \times 30 \mathrm{~m} \\
\text { repeated sprints } \\
\text { with } 30 \mathrm{~s} \text { rest }\end{array}$ & $\begin{array}{c}\text { Montmorency tart cherry } \\
\text { juice } \\
\text { Per } 1000 \mathrm{~mL} \\
73.5 \mathrm{mg} \\
\text { cyanidin-3-glucoside } \\
\text { (HPLC) } \\
\text { 178.8 mg of GAE (total } \\
\text { phenols) } \\
0.58 \text { trolox equivalent } \\
\text { (TEAC) } \\
\text { Based on previous work } \\
\text { from own laboratory (61). }\end{array}$ & $\begin{array}{c}2 \times 30 \mathrm{~mL} \\
\text { concentrate for } \\
8 \text { days ( } 4 \text { days } \\
\text { pre-exercise, } \\
\text { on the day of, } \\
\text { and } 3 \text { days } \\
\text { post-exercise) }\end{array}$ & $\begin{array}{c}\text { MIVC (PE, 24, 48, 72) } \\
\text { DOMS (PE, 24, 48, 72) } \\
\text { CMJ (PE, 24, 48, 72) } \\
\text { CK (PE, 24, 48, 72) } \\
\text { CRP, (PE, 24, 48, 72) }\end{array}$ \\
\hline $\begin{array}{l}\text { Clifford } \\
\text { et al. } \\
\text { 2016a [13] }\end{array}$ & $\begin{array}{c}\text { Male collegiate team } \\
\text { sport players } \\
\text { Age } 22 \pm 2.5 \text { years } \\
\text { Height } 1.80 \pm 0.70 \mathrm{~m} \\
\text { Mass } 75.1 \pm 10.9 \mathrm{~kg} \\
n=20\end{array}$ & Parallel & $\begin{array}{c}20 \times 30 \mathrm{~m} \\
\text { sprints on day } 1 \\
\text { and day } 4(72 \mathrm{~h} \\
\text { apart) }\end{array}$ & $\begin{array}{c}\text { Beetroot juice } \\
\text { Per } 1000 \mathrm{~mL} \\
1606.9 \pm 151 \mathrm{mg} \mathrm{GAE} \\
\text { (total phenols) } \\
11.4 \pm 0.2 \mathrm{mmol} \text { trolox } \\
\text { equivalents (TEAC) (62). }\end{array}$ & $\begin{array}{l}2 \times 250 \mathrm{~mL} \text { for } \\
3 \text { days (day of } \\
\text { and } 2 \text { days } \\
\text { post-exercise) }\end{array}$ & $\begin{array}{c}\text { MIVC (PE, 24, 48, 72, 96) } \\
\text { CMJ (PE, 24, 48, 72, 96) } \\
\text { CK (PE, 24, 48, 72, 96) } \\
\text { CRP (PE, 24, 48, 72, 96) } \\
\text { PC (PE, 24, 48, 72, 96) }\end{array}$ \\
\hline
\end{tabular}


Table 1. Cont.

\begin{tabular}{|c|c|c|c|c|c|c|}
\hline Authors & $\begin{array}{c}\text { Participant } \\
\text { Characteristics at } \\
\text { Baseline }\end{array}$ & $\begin{array}{l}\text { Study } \\
\text { Design }\end{array}$ & $\begin{array}{c}\text { Exercise } \\
\text { Intervention }\end{array}$ & Polyphenol Supplement & $\begin{array}{l}\text { Dosage and } \\
\text { Duration }\end{array}$ & $\begin{array}{c}\text { Outcome Variables and } \\
\text { Time of Measurement } \\
\text { (h) }\end{array}$ \\
\hline $\begin{array}{c}\text { Clifford } \\
\text { et al. } \\
\text { 2016b [27] }\end{array}$ & $\begin{array}{l}\text { Male recreational } \\
\text { active participants } \\
\text { Age } 21.3 \pm 4 \text { years } \\
\text { Height } 178 \pm 0.76 \mathrm{~m} \\
\text { Mass } 75.6 \pm 8.3 \mathrm{~kg} \\
\quad n=30\end{array}$ & Parallel & $\begin{array}{l}5 \times 20 \text { drop } \\
\text { jumps }\end{array}$ & $\begin{array}{l}\text { Beetroot juice (high } 250 \\
\text { mL vs. low dose } 125 \mathrm{~mL} \text { ) } \\
\text { Per } 250 \mathrm{~mL} \\
401.72 \pm 37.72 \mathrm{mg} \mathrm{GAE} \\
\text { (total phenols) } \\
2.85 \pm 0.05 \text { mmol trolox } \\
\text { equivalents (DPPH) }\end{array}$ & $\begin{array}{l}3 \times \text { servings } \\
\text { on day of } \\
\text { exercise and } 2 \\
\times \text { servings for } \\
\text { two days } \\
\text { post-exercise }\end{array}$ & $\begin{array}{c}\text { MIVC, }(\mathrm{PE}, 24,48,72) \\
\text { CMJ (PE, 24 48, 72) } \\
\text { CK (PE, 24, 48, 72) } \\
\text { IL-6 (PE, 24, 48, 72) }\end{array}$ \\
\hline $\begin{array}{l}\text { Clifford } \\
\text { et al. } \\
\text { 2017a [35] }\end{array}$ & $\begin{array}{l}\text { Recreational runners } \\
\text { Age } 40.5 \pm 11 \text { years } \\
\text { Height } 1.71 \pm 0.08 \mathrm{~cm} \\
\text { Mass } 70.3 \pm 10.85 \mathrm{~kg} \\
n=34(\mathrm{~m}=21 ; \mathrm{f}=13)\end{array}$ & Parallel & $\begin{array}{l}\text { Druridge Bay } \\
\text { Marathon } \\
\text { (Northumbria, } \\
\text { UK) }\end{array}$ & $\begin{array}{c}\text { Beetroot juice }(250 \mathrm{~mL}) \\
\text { Per } 250 \mathrm{~mL} \\
\sim 400 \text { mg GAE (total } \\
\text { phenols) } \\
\sim 3 \text { mmol trolox } \\
\text { equivalents (DPPH) (62). }\end{array}$ & $\begin{array}{l}3 \times \text { servings } \\
\text { on day of } \\
\text { exercise and } 2 \\
\times \text { servings for } \\
\text { two days } \\
\text { post-exercise }\end{array}$ & $\begin{array}{c}\text { MIVC (PE, 24, 48) } \\
\text { DOMS (PE, 24, 48) } \\
\text { CMJ (PE, 24, 48) } \\
\text { CK (PE, 24, 48) } \\
\text { CRP (PE, 24, 48) } \\
\text { IL-6 (PE, 24, 48) }\end{array}$ \\
\hline $\begin{array}{c}\text { Clifford } \\
\text { et al. } \\
\text { 2017b [42] }\end{array}$ & $\begin{array}{c}\text { Male healthy } \\
\text { untrained participants } \\
\text { Age } 21.7 \pm 2.3 \text { years } \\
\text { Height } 178.0 \pm 6.46 \\
\mathrm{~cm} \\
\text { Mass } 75.1 \pm 10.13 \mathrm{~kg} \\
n=30\end{array}$ & Parallel & $\begin{array}{l}5 \times 20 \text { drop } \\
\text { jumps }\end{array}$ & $\begin{array}{c}\text { Beetroot juice }(250 \mathrm{~mL}) \\
\text { Per } 250 \mathrm{~mL} \\
\sim 400 \text { mg GAE (total } \\
\text { phenols) (62). }\end{array}$ & $\begin{array}{l}3 \times \text { servings } \\
\text { on day of } \\
\text { exercise and } 2 \\
\times \text { servings for } \\
\text { two days } \\
\text { post-exercise }\end{array}$ & $\begin{array}{c}\text { MIVC (PE, 24, 48, 72) } \\
\text { CMJ (PE, 24, 48, 72) } \\
\text { CK, (PE, 24, 48, 72) } \\
\text { CRP (PE, 24, 48, 72) }\end{array}$ \\
\hline $\begin{array}{l}\text { Connoll, } \\
\text { McHugh } \\
\text { and } \\
\text { Padilla } \\
\text { Zakour } \\
2006(9)\end{array}$ & $\begin{array}{c}\text { Male } \\
\text { Age } 22 \pm 4 \text { y ears } \\
\text { Height } 1.78 \pm 0.86 \mathrm{~m} \\
\text { Mass } 90 \pm 18 \mathrm{~kg} \\
n=16\end{array}$ & Crossover & $\begin{array}{c}2 \times 20 \text { eccentric } \\
\text { elbow } \\
\text { contractions }\end{array}$ & $\begin{array}{l}\text { Tart cherry juice and } \\
\text { apple juice blend } \\
\text { Per } 12 \mathrm{fl} \mathrm{oz} \\
600 \text { mg of GAE (total } \\
\text { phenols) } \\
40 \text { mg of } \\
\text { cyanidin-3-glucoside } \\
\text { equivalents (pH } \\
\text { differential) }\end{array}$ & $\begin{array}{l}2 \times 12 \mathrm{fl} \mathrm{oz} \\
\text { bottles for } 8 \\
\text { days ( } 3 \text { days } \\
\text { pre-exercise, } \\
\text { on the day of, } \\
\text { and } 4 \text { days } \\
\text { post-exercise) }\end{array}$ & $\begin{array}{c}\text { MIVC }(24,48,72,96) \\
\text { DOMS, }(24,48,72,96)\end{array}$ \\
\hline $\begin{array}{l}\text { Daab et al. } \\
2020 \text { [43] }\end{array}$ & $\begin{array}{c}\text { Male } \\
\text { semi-professional } \\
\text { soccer players } \\
\text { Age } 22.1 \pm 0.56 \text { years } \\
\text { Height } 178 \pm 1.19 \mathrm{~cm} \\
\text { Mass } 75.8 \pm 5.58 \mathrm{~kg} \\
n=13\end{array}$ & Crossover & $\begin{array}{l}\text { Loughborough } \\
\text { intermittent } \\
\text { shuttle test }\end{array}$ & $\begin{array}{c}\text { Beetroot juice } \\
\text { Polyphenol content not } \\
\text { stated }\end{array}$ & $\begin{array}{l}2 \times 150 \mathrm{~mL} \\
\text { per day for } 7 \\
\text { days (3 days } \\
\text { pre-exercise, } \\
\text { on the day of, } \\
\text { and } 3 \text { days } \\
\text { post-exercise) }\end{array}$ & $\begin{array}{c}\text { MIVC (PE, 24, 48, 72) } \\
\text { DOMS (PE, 24, 48, 72) } \\
\text { CMJ (PE, 24, 48, 72) } \\
\text { CK (PE, 24, 48, 72) } \\
\text { CRP (PE, 24, 48, 72) }\end{array}$ \\
\hline $\begin{array}{c}\text { Howatson } \\
\text { et al. } 2010 \\
{[36]}\end{array}$ & $\begin{array}{l}\text { Marathon runners } \\
\text { Age } 37.5 \pm 9 \text { years } \\
\text { Height } 1.76 \pm 0.07 \mathrm{~m} \\
\text { Mass } 73.3 \pm 9.85 \mathrm{~kg} \\
n=20(\mathrm{~m}=13 ; \mathrm{f}=7)\end{array}$ & Parallel & $\begin{array}{c}\text { London } \\
\text { Marathon (UK) }\end{array}$ & $\begin{array}{l}\text { Tart cherry juice and } \\
\text { apple juice blend } \\
55 \mathrm{mmol} \cdot \mathrm{L}^{-1} \text { trolox } \\
\text { equivalents (ORAC) } \\
\text { Per } 12 \mathrm{fl} \mathrm{oz} \\
600 \mathrm{mg} \text { of GAE (total } \\
\text { phenols) } \\
40 \mathrm{mg} \text { of } \\
\text { cyanidin-3-glucoside } \\
\text { equivalents (pH } \\
\text { differential) (9). }\end{array}$ & $\begin{array}{l}2 \times 8 \mathrm{fl} \mathrm{oz} \\
\text { bottles for } 8 \\
\text { days ( } 5 \text { days } \\
\text { pre-exercise, } \\
\text { on the day of, } \\
\text { and } 2 \text { days } \\
\text { post-exercise) }\end{array}$ & $\begin{array}{c}\text { MIVC (PE, 24, 48) } \\
\text { DOMS (PE, 24,48) } \\
\text { CK (PE, 24, 48) } \\
\text { CRP (PE, 24, 48) } \\
\text { IL-6 (PE, 24 48) } \\
\text { PC (PE, 24, 48) }\end{array}$ \\
\hline $\begin{array}{c}\text { Hutchison } \\
\text { et al. } 2016 \\
{[44]}\end{array}$ & $\begin{array}{l}\text { Healthy participants } \\
\text { Age } 20.2 \pm 0.6 \text { years } \\
\text { Height } 165.75 \pm 3 \mathrm{~cm} \\
\text { Mass } 64.2 \pm 5 \mathrm{~kg} \\
n=16(\mathrm{~m}=3 ; \mathrm{f}=13)\end{array}$ & Parallel & $\begin{array}{c}3 \times 10 \text { eccentric } \\
\text { squats }\end{array}$ & $\begin{array}{l}\text { Blackcurrant nectar } \\
7340 \mu \text { mol trolox } \\
\text { equivalents (TEAC) } \\
\text { Per } 16 \text { fl oz } \\
193.25 \text { mg malvidin } \\
\text { glucosides (pH } \\
\text { differential) } \\
175.69 \text { mg cyanidin } \\
\text { glucosides (pH } \\
\text { differential) }\end{array}$ & $\begin{array}{l}16 \mathrm{fl} \mathrm{oz} \text { bottle } \\
\text { twice per day } \\
\text { for } 8 \text { days ( } 4 \\
\text { days } \\
\text { pre-exercise, } \\
\text { on the day of, } \\
\text { and } 3 \text { days } \\
\text { post-exercise) }\end{array}$ & $\begin{array}{c}\text { DOMS (PE, 24, 48, 96) } \\
\text { CK (PE, 24, 48, 96) } \\
\text { IL-6 (PE, 24, 48, 96) }\end{array}$ \\
\hline
\end{tabular}


Table 1. Cont.

\begin{tabular}{|c|c|c|c|c|c|c|}
\hline Authors & $\begin{array}{c}\text { Participant } \\
\text { Characteristics at } \\
\text { Baseline }\end{array}$ & $\begin{array}{l}\text { Study } \\
\text { Design }\end{array}$ & $\begin{array}{c}\text { Exercise } \\
\text { Intervention }\end{array}$ & Polyphenol Supplement & $\begin{array}{l}\text { Dosage and } \\
\text { Duration }\end{array}$ & $\begin{array}{l}\text { Outcome Variables and } \\
\text { Time of Measurement } \\
\text { (h) }\end{array}$ \\
\hline $\begin{array}{c}\text { Kuehl } \\
\text { et al. 2010 } \\
\text { [37] }\end{array}$ & $\begin{array}{c}\text { Healthy runners } \\
\text { Age } 35.8 \pm 9.6 \text { years } \\
n=54(\mathrm{~m}=36 ; \mathrm{f}=18)\end{array}$ & Parallel & $\begin{array}{l}\text { Oregon Hood to } \\
\text { Coast Relay Race } \\
\text { (USA) }\end{array}$ & $\begin{array}{c}\text { Montmorency tart cherry } \\
\text { juice and apple juice blend } \\
\text { Per } 12 \mathrm{fl} \mathrm{oz} \\
600 \text { mg of GAE (total } \\
\text { phenols) } \\
40 \text { mg of } \\
\text { cyanidin-3-glucoside } \\
\text { equivalents (pH } \\
\text { differential) (9). }\end{array}$ & $\begin{array}{c}2 \times 355 \mathrm{~mL} \\
\text { tart cherry } \\
\text { juice for } 8 \text { days } \\
\text { (7 days } \\
\text { pre-exercise, } \\
\text { on the day of } \\
\text { the trial) }\end{array}$ & DOMS (PE) \\
\hline $\begin{array}{l}\text { Kupsaravic, } \\
\text { McShane } \\
\text { and } \\
\text { Clifford. } \\
2019 \text { [45] }\end{array}$ & $\begin{array}{c}\text { Elite male rugby } \\
\text { union players } \\
\text { Age } 28 \pm 4 \text { years } \\
\text { Height } 1.88 \pm 0.64 \mathrm{~m} \\
\text { Mass } 106.8 \pm 7.6 \mathrm{~kg} \\
n=10\end{array}$ & Crossover & $\begin{array}{l}\text { Rugby Union } \\
\text { match }\end{array}$ & $\begin{array}{c}\text { Montmorency tart cherry } \\
\text { juice } \\
\text { Polyphenol content not } \\
\text { stated }\end{array}$ & $\begin{array}{c}2 \times 30 \mathrm{~mL} \\
\text { concentrate for } \\
5 \text { days }(2 \text { days } \\
\text { pre-exercise, } \\
\text { on the day of, } \\
2 \text { days } \\
\text { post-exercise) }\end{array}$ & DOMS $(24,48,72)$ \\
\hline $\begin{array}{l}\text { Lamb et al. } \\
2019 \text { [28] }\end{array}$ & $\begin{array}{c}\text { Male non-resistance } \\
\text { trained } \\
\text { Age } 24 \text { IQR } 22,33 \\
\text { years } \\
\text { BMI 25.6 } \pm 4 \mathrm{~kg} \mathrm{~m}^{-2} \\
n=36\end{array}$ & Parallel & $\begin{array}{l}5 \times 10 \text { eccentric } \\
\text { elbow } \\
\text { contractions non } \\
\text { dominant arm }\end{array}$ & $\begin{array}{l}\text { Montmorency tart cherry } \\
\text { juice and Pomegranate } \\
\text { Wonderful juice } \\
\text { Tart cherry juice per } 30 \mathrm{~mL} \\
294.7 \pm 14.9 \mathrm{mg} \mathrm{GAE} \\
\text { (total phenols) } \\
7.7 \pm 0.3 \mathrm{mg} \text { anthocyanins } \\
\text { (pH differential) } \\
\text { Pomegranate Wonderful } \\
\text { per } 250 \mathrm{~mL} \\
878.9 \pm 92.7 \mathrm{mg} \mathrm{GAE} \\
\text { (total phenols) } \\
49.4 \pm 2.0 \mathrm{mg} \text { total } \\
\text { anthocyanins (pH } \\
\text { differential) }\end{array}$ & $\begin{array}{c}2 \times 30 \mathrm{~mL} \text { of } \\
\text { concentrate for } \\
9 \text { days (cherry) } \\
\text { and } 2 \times 250 \\
\text { mL for } 9 \text { days } \\
\text { (pomegranate) } \\
(4 \text { days } \\
\text { pre-exercise, } \\
\text { on the day of, } \\
4 \text { days } \\
\text { post-exercise) }\end{array}$ & $\begin{array}{c}\text { MIVC (PE, 24, 48, 72, 96) } \\
\text { DOMS (PE, 24, 48, 72, 96) } \\
\text { CK (PE, 24, 48, 72, 96) }\end{array}$ \\
\hline $\begin{array}{l}\text { Lima et al. } \\
2019 \text { [46] }\end{array}$ & $\begin{array}{c}\text { Healthy male physical } \\
\text { education students } \\
\text { Age } 22.3 \pm 2.6 \text { years } \\
\text { Height } 176.6 \pm 6.4 \mathrm{~cm} \\
\text { Mass } 77.1 \pm 10.5 \mathrm{~kg} \\
n=30\end{array}$ & Parallel & $\begin{array}{l}30 \text { min downhill } \\
\text { run at } 70 \% \\
\dot{V} \mathrm{O}_{2 \max }\end{array}$ & $\begin{array}{c}\text { Anthocyanin-rich } \\
\text { antioxidant juice that } \\
\text { consisted of a mixture of } \\
\text { clarified apple juice with } \\
\text { plum, blueberry, } \\
\text { maquiberry, raspberry } \\
\text { and cranberry } \\
67,680 \mu \mathrm{mol} \cdot \mathrm{mL}^{-1} \text { of } \\
\text { trolox equivalents (ORAC) } \\
\text { Per } 240 \mathrm{~mL} \\
58 \mathrm{mg} \text { of anthocyanins }\end{array}$ & $\begin{array}{c}2 \times 240 \mathrm{~mL} \text { for } \\
9 \text { days ( } 4 \text { days } \\
\text { pre-exercise, } \\
\text { on the day of, } \\
4 \text { days } \\
\text { post-exercise) }\end{array}$ & $\begin{array}{c}\text { MIVC (PE, 24, 48, 72. 96) } \\
\text { DOMS }(24,48,72,96) \\
\text { CK }(48,96)\end{array}$ \\
\hline $\begin{array}{l}\text { Lynn et al. } \\
2018 \text { [11] }\end{array}$ & $\begin{array}{c}\text { Recreational runners } \\
\text { Age } 30.9 \pm 10.53 \\
\text { years } \\
\text { Height } 1.74 \pm 0.08 \mathrm{~m} \\
\text { Mass } 71.4 \pm 10.5 \mathrm{~kg} \\
\text { BMI } 23.5 \pm 2.45 \mathrm{~kg} \\
\mathrm{~m}^{-2} \\
n=21(\mathrm{~m}=16 ; \mathrm{f}=5)\end{array}$ & Parallel & $\begin{array}{l}\text { Sheffield Half } \\
\text { Marathon (UK) }\end{array}$ & $\begin{array}{c}\text { Bilberry juice } \\
\text { Per } 200 \mathrm{~mL} \\
744.14 \pm 81.75 \mathrm{mg} \text { of GAE } \\
\text { (total phenols) } \\
80.04 \pm 3.51 \mathrm{mg} \text { of total } \\
\text { anthocyanins (pH } \\
\text { differential) }\end{array}$ & $\begin{array}{c}2 \times 200 \mathrm{~mL} \text { for } \\
8 \text { days ( } 5 \text { days } \\
\text { pre-exercise, } \\
\text { on the day of, } \\
2 \text { days } \\
\text { post-exercise) }\end{array}$ & $\begin{array}{c}\text { DOMS (PE, 24, 48) } \\
\text { CK (PE, 24, 48) } \\
\text { CRP (PE, 24, 48) }\end{array}$ \\
\hline $\begin{array}{l}\text { Machin } \\
\text { et al. } 2014 \\
{[25]}\end{array}$ & $\begin{array}{c}\text { Male non-resistance } \\
\text { trained } \\
\text { Age } 22.3 \pm 4.1 \text { years } \\
\text { Height } 174.9 \pm 6.2 \mathrm{~cm} \\
\text { Mass } 73.8 \pm 11.5 \mathrm{~kg} \\
n=45\end{array}$ & Parallel & $\begin{array}{l}20 \text { min of } \\
\text { downhill } \\
\text { running and } 40 \\
\text { repetitions of } \\
\text { bilateral } \\
\text { eccentric elbow } \\
\text { contractions }\end{array}$ & $\begin{array}{c}\text { Pomegranate Wonderful } \\
\text { juice (high } 2 \times 30 \mathrm{~mL} \text { vs. } \\
\text { low } 1 \times 30 \mathrm{~mL}) \\
\text { Per } 30 \mathrm{~mL} \\
650 \mathrm{mg} \text { of GAE consisting } \\
\text { of } 95.5 \% \text { ellagitannins, } \\
3.5 \% \text { ellagic acid, and } 1 \% \\
\text { anthocyanins }\end{array}$ & $\begin{array}{l}1 \text { or } 2 \times \\
\text { servings for } 8 \\
\text { days ( } 3 \text { days } \\
\text { pre-exercise, } \\
\text { on the day of, } \\
4 \text { days } \\
\text { post-exercise) }\end{array}$ & $\begin{array}{l}\text { MIVC (PE, 24, 48, 72, 96) } \\
\text { DOMS (PE, 24, 48, 72, 96) }\end{array}$ \\
\hline
\end{tabular}


Table 1. Cont.

\begin{tabular}{|c|c|c|c|c|c|c|}
\hline Authors & $\begin{array}{c}\text { Participant } \\
\text { Characteristics at } \\
\text { Baseline }\end{array}$ & $\begin{array}{l}\text { Study } \\
\text { Design }\end{array}$ & $\begin{array}{c}\text { Exercise } \\
\text { Intervention }\end{array}$ & Polyphenol Supplement & $\begin{array}{l}\text { Dosage and } \\
\text { Duration }\end{array}$ & $\begin{array}{c}\text { Outcome } \\
\text { Variables and } \\
\text { Time of } \\
\text { Measurement (h) }\end{array}$ \\
\hline $\begin{array}{c}\text { McLeay } \\
\text { et al. } 2012 \\
{[12]}\end{array}$ & $\begin{array}{l}\text { Healthy recreational } \\
\text { females } \\
\text { Age } 22 \pm 1 \text { years } \\
\text { Height } 167 \pm 5 \mathrm{~cm} \\
\text { Mass } 62 \pm 8 \mathrm{~kg} \\
n=14\end{array}$ & Crossover & $\begin{array}{l}3 \times 100 \text { eccentric } \\
\text { knee extensions }\end{array}$ & $\begin{array}{l}\text { Smoothie with New } \\
\text { Zealand blueberries }(200 \\
\text { g), banana }(\sim 50 \mathrm{~g}) \text { and } \\
\text { apple juice }(200 \mathrm{~mL}) \\
5417 \mu \mathrm{mol} \text { trolox } \\
\text { equivalents (ORAC) } \\
\text { Per } 100 \mathrm{~mL} \\
168 \mathrm{mg} \text { of GAE (total } \\
\text { phenols) } \\
96.6 \mathrm{mg} \text { of anthocyanins } \\
26 \mathrm{mg} \text { of phenolic acid } \\
10.2 \mathrm{mg} \text { of flavonoids }\end{array}$ & $\begin{array}{l}3 \times \text { servings on } \\
\text { the day of } \\
\text { exercise and } 1 \times \\
\text { serving for } 2 \text { days } \\
\text { post-exercise }\end{array}$ & $\begin{array}{c}\text { MIVC }(12,36,60) \\
\text { DOMS }(12,36,60) \\
\text { CK }(12,36,60) \\
\text { IL-6 }(12,36,60) \\
\text { PC }(12,36,60)\end{array}$ \\
\hline $\begin{array}{c}\text { Morehen } \\
\text { et al. } 2020 \\
\text { [47] }\end{array}$ & $\begin{array}{c}\text { Male Professional } \\
\text { Rugby players } \\
\text { Age } 18 \pm 1 \text { years } \\
\text { Height } 182 \pm 0.04 \mathrm{~cm} \\
\text { Mass } 92.2 \pm 8.6 \mathrm{~kg} \\
n=11\end{array}$ & Crossover & Rugby Union match & $\begin{array}{c}\text { Montmorency tart cherry } \\
\text { juice } \\
\text { Per } 30 \mathrm{~mL} \\
320 \mathrm{mg} \text { of anthocyanins }\end{array}$ & $\begin{array}{l}2 \times 30 \mathrm{~mL} \\
\text { concentrate per } \\
\text { day for } 7 \text { days, }(4 \\
\text { days pre-match, } \\
\text { on the day of, } 2 \\
\text { days post-match) }\end{array}$ & $\begin{array}{c}\text { DOMS }(24,48) \\
\text { CMJ }(48) \\
\text { IL-6 (PE, 48) }\end{array}$ \\
\hline $\begin{array}{c}\text { Morgan } \\
\text { et al. } 2018 \\
{[14]}\end{array}$ & $\begin{array}{c}\text { Healthy recreational } \\
\text { active males } \\
\text { Age } 22.8 \pm 3.3 \text { years } \\
\text { Height } 1.84 \pm 0.59 \mathrm{~cm} \\
\text { Mass } 85.3 \pm 12 \mathrm{~kg} \\
n=10\end{array}$ & Crossover & $\begin{array}{l}\text { Single leg extension } \\
10 \times 10 \text { repetitions } \\
\text { at } 80 \% 1 \mathrm{RM}\end{array}$ & $\begin{array}{c}\text { Ecuadorian cacao juice } \\
\left(\text { ZumaCacao }{ }^{\circledR}\right) \\
\text { Per } 330 \text { mL serving } \\
154 \text { mg of polyphenols } \\
8 \text { mg epicatechin } \\
43 \text { mg catechins } \\
23 \text { mg flavanols } \\
12 \text { mg proanthocyanidins } \\
\text { HPLC -Atlas, Bioscience, } \\
\text { Inc, Tucson, Arizona, USA }\end{array}$ & $\begin{array}{c}330 \mathrm{~mL} \text { per day } \\
\text { for } 10 \text { days ( } 7 \\
\text { days pre-exercise, } \\
\text { on the day of, } 2 \\
\text { days } \\
\text { post-exercise) }\end{array}$ & $\begin{array}{c}\text { MIVC (PE, 24, 48) } \\
\text { DOMS (PE, 24, 48) } \\
\text { CMJ (PE, 24, 48) } \\
\text { CK (PE, 24, 48) } \\
\text { CRP (PE, 24, 48) } \\
\text { PC (PE, 24, 48) }\end{array}$ \\
\hline $\begin{array}{c}\text { Peschek } \\
\text { et al. } 2014 \\
\text { [26] }\end{array}$ & $\begin{array}{c}\text { Male well trained } \\
\text { runners and } \\
\text { triathletes } \\
\text { Age } 24.6 \pm 5.6 \text { years } \\
\text { Height } 182.1 \pm 6.3 \mathrm{~cm} \\
\text { Mass } 73.4 \pm 7 \mathrm{~kg} \\
\text { Body fat percentage } \\
13.7 \pm 5.1 \% \\
n=8\end{array}$ & Crossover & $\begin{array}{l}30 \text { min downhill } \\
\text { run at } 70 \% \dot{V} \mathrm{O}_{2 \max }\end{array}$ & $\begin{array}{l}\text { Unsweetened Cocoa Via } \\
\text { consisted of cocoa powder, } \\
\text { salt, and soy lecithin } \\
\text { Per } 240 \mathrm{~mL} \\
350 \mathrm{mg} \text { flavanols }\end{array}$ & $\begin{array}{l}1 \mathrm{~g} \cdot \mathrm{kg}^{-1} \text { of body } \\
\text { weight of cocoa } \\
\text { milk at } 1 \mathrm{~h} \text { and at } \\
2 \mathrm{~h} \text { post-exercise }\end{array}$ & $\begin{array}{c}\text { MIVC }(24,48) \\
\text { DOMS }(24,48) \\
\text { CK }(24,48)\end{array}$ \\
\hline $\begin{array}{l}\text { Quinlan } \\
\text { and Hill } \\
2020[48]\end{array}$ & $\begin{array}{c}\text { Team sport } \\
\text { recreational athletes } \\
\text { Age } 26.5 \pm 4.5 \text { years } \\
\text { Height } 175.3 \pm 9.75 \\
\mathrm{~cm} \\
\text { Mass } 70.2 \pm 12.85 \mathrm{~kg} \\
\text { Predicted } \dot{V} \mathrm{O}_{2 \text { peak }} \\
44.4 \pm 8.1 \\
\mathrm{~mL} \cdot \mathrm{kg}^{-1} \cdot \mathrm{min}^{-1} \\
n=20(\mathrm{~m}=8 ; \mathrm{f}=12)\end{array}$ & Parallel & $\begin{array}{l}\text { Loughborough } \\
\text { intermittent shuttle } \\
\text { test followed by } 12 \\
\times 20 \text { m sprints }\end{array}$ & $\begin{array}{l}\text { Montmorency tart cherry } \\
\text { juice } \\
\text { Polyphenol content not } \\
\text { stated }\end{array}$ & $\begin{array}{c}2 \times 30 \mathrm{~mL} \\
\text { concentrate for } 8 \\
\text { days ( } 5 \text { day } \\
\text { pre-exercise, on } \\
\text { the day of, } 2 \text { days } \\
\text { post-exercise) }\end{array}$ & $\begin{array}{c}\text { MIVC (PE, 24, 48) } \\
\text { DOMS (PE, 24, 48) } \\
\text { CMJ (PE, 24, 48) } \\
\text { CK (PE, 24, 48) } \\
\text { CRP (PE, 24, 48) }\end{array}$ \\
\hline $\begin{array}{c}\text { Trombold } \\
\text { et al. } 2011 \\
{[10]}\end{array}$ & $\begin{array}{c}\text { Male recreational } \\
\text { active } \\
\text { Age } 21.9 \pm 2.4 \text { years } \\
\text { Height } 179.1 \pm 8.4 \mathrm{~cm} \\
\text { Mass } 80.2 \pm 7.5 \mathrm{~kg} \\
n=17\end{array}$ & Crossover & $\begin{array}{l}3 \times 20 \text { eccentric } \\
\text { elbow contractions, } \\
6 \times 10 \text { eccentric } \\
\text { knee contractions }\end{array}$ & $\begin{array}{c}\text { Pomegranate Wonderful } \\
\text { juice } \\
\text { Per } 1000 \mathrm{~mL} \\
1979 \mathrm{mg} \text { of tannins } \\
384 \mathrm{mg} \text { of anthocyanins } \\
121 \mathrm{mg} \text { of ellagic acid } \\
\text { derivatives } \\
\text { (Content obtained from } \\
\text { the manufacturer) }\end{array}$ & $\begin{array}{c}2 \times 250 \mathrm{~mL} \text { for } 15 \\
\text { days ( } 8 \text { days } \\
\text { pre-exercise, on } \\
\text { the day of, } 6 \text { days } \\
\text { post-exercise) }\end{array}$ & $\begin{array}{l}\text { MIVC (PE, 24, 48, } \\
72,96) \\
\text { DOMS, (PE, 24, 48, } \\
72,96)\end{array}$ \\
\hline
\end{tabular}

BMI, body mass index; CK, creatine kinase; CMJ, countermovement jump; CRP, c-reactive protein; DOMS, delayed onset muscle soreness; DPPH, 2,2-diphenyl-1-picrylhydrazyl; GAE, gallic acid equivalents; h, hours; HPLC, high performance liquid chromatography; IQR, interquartile range; IL-6, interleukin-6; MIVC, maximal isometric voluntary contraction; ORAC, oxygen radical absorbance capacity; PC, protein carbonyls; $\mathrm{PE}$, post-exercise; $\mathrm{RM}$, repetition maximum; TEAC, trolox equivalent antioxidant capacity; $\dot{V} \mathrm{O}_{2 \max }$, maximal oxygen uptake; $\dot{V} \mathrm{O}_{2 \text { peak }}$, peak oxygen uptake. 


\subsubsection{Participants}

A total of 527 participants (male $n=425$; female $n=102$ ) were included in this metaanalysis. Of the 527 participants, 29 (5.5\%) were semi-professional athletes [40,43], 31 (5.8\%) were professional athletes [38,45,47] and $467(88.6 \%)$ participants were recreationally trained.

\subsubsection{Intervention}

All of the included studies compared a polyphenol-rich food, juice or concentrate to a placebo as follows:

- $\quad$ cherry $(n=12) *[9,28,34,36-41,45,47,48]$

- $\quad$ pomegranate $(n=3) *[10,25,28]$

- $\quad$ beetroot $(n=5)[13,27,35,42,43]$

- $\operatorname{cocoa}(n=2)[14,26]$

- $\quad$ bilberry $(n=1)[11]$

- blueberry $(n=1)[12]$

- $\quad$ blackcurrant $(n=1)$ [44]

- $\quad$ mixed fruit juice $(n=1)$ [46]

* One study included cherry and pomegranate [28].

Trials were conducted in the UK $(n=16)$, the USA $(n=6)$, New Zealand $(n=1)$, Tunisia $(n=1)$, and Brazil $(n=1)$.

\subsubsection{Adverse Events}

Only two of 25 studies asked participants about adverse responses to the supplements $[11,12]$; both reported no adverse events.

\subsection{Outcomes}

\subsubsection{Primary}

Of the 25 studies included in this review, 19 reported on MIVC, 24 reported a measure of muscle soreness, and 11 reported on CMJ (see Table 1). There was, however, variability between the studies in the number of time points measured ranging from immediately post-exercise to $96 \mathrm{~h}$ post-exercise.

\subsubsection{Secondary}

Of the 25 studies included in this review, 18 reported on CK, 12 on CRP, eight on IL-6 and five measured PC (see Table 1). There was substantial variation in the number of post-exercise time points measured by each individual study. CK and CRP were measured up to $96 \mathrm{~h}$ post-exercise in some studies, however, IL-6 and PC were only measured for a maximum of $72 \mathrm{~h}$ post-exercise.

\subsection{Risk of Bias within Studies}

Of the 25 studies, 15 were rated as some concerns and 10 as high risk of bias (see Figure 2). Factors driving an overall high risk of bias were lack of information in the manuscript, failure to blind assessors, issues with randomisation, and lack of information on adherence to the intervention. An issue with the studies included in this review was that none pre-registered their study protocol so there was no way of confirming whether they had a pre-specified data analysis plan [24]. Visual inspection of the funnel plots (see Supplementary File 2) did not identify substantial asymmetry providing little evidence of publication bias.

\subsection{Syntheses of Results \\ 3.5.1. Primary Outcomes MIVC}

Polyphenol supplementation caused small but significant increases in MIVC in comparison to placebo immediately post-exercise $(\mathrm{SMD}=0.23,95 \%$ CI $0.04,0.42 ; p=0.02$; 
participants $=370 ;$ studies $=14 ; \mathrm{I}^{2}=0 \%$; low-quality evidence), $24 \mathrm{~h}$ post-exercise $(\mathrm{SMD}=0.39$, $95 \%$ CI 0.15, 0.62; $p=0.001$; participants $=466$; studies $=19 ; \mathrm{I}^{2}=48 \%$; low-quality evidence), $48 \mathrm{~h}$ post-exercise $(\mathrm{SMD}=0.48,95 \%$ CI $0.28,0.67 ; p<0.001$; participants $=466$; studies $=19 ; \mathrm{I}^{2}=30 \%$; moderate-quality evidence), $72 \mathrm{~h}$ post-exercise (SMD $=0.29,95 \% \mathrm{CI} 0.11$, $0.46 ; p=0.001$; participants $=338$; studies $=13 ; \mathrm{I}^{2}=0 \%$; low-quality evidence) and at $96 \mathrm{~h}$ post-exercise $(\mathrm{SMD}=0.50,95 \% \mathrm{CI} 0.16,0.83 ; p=0.004$; participants $=170$; studies $=5$; $\mathrm{I}^{2}=45 \%$; very low-quality evidence). These SMDs equated to improvements in MIVC of $6.0 \%$ (immediately post), 7.7\% (24 h), 9.6\% (48 h), 5.7\% (72 h) and 13.0\% (96h) (see Figure 3).

\begin{tabular}{|c|c|c|c|c|c|c|c|c|c|c|c|}
\hline Study ID & Study Design & Experimental & Comparator & Outcome & 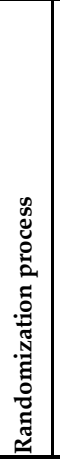 & 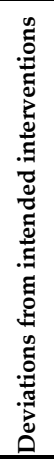 & 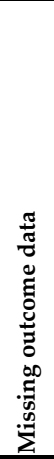 & 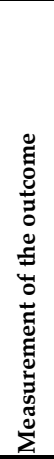 & 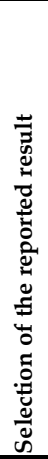 & $\begin{array}{l}\overline{\widetilde{J}} \\
\text { ठे }\end{array}$ & \\
\hline A bbotetal2019 [38] & Cross-over & T art cherry & P lacebo & D $0 M \mathrm{~S}$ & + & + & + & + & $?$ & (3) & \\
\hline Bow telletal. 2011 [34] & Cross-over & T art cherry & P lacebo & M IV C & $?$ & $?$ & + & + & $?$ & (3) & \\
\hline \begin{tabular}{|l|l} 
C onnolly etal. 2006 [9] \\
\end{tabular} & C ross-over & T art cherry/ap p le b lend & P lacebo & M IV C & + & $\theta$ & + & $?$ & $?$ & $\Theta$ & \\
\hline D aab etal. 2020 [43] & Cross-over & B eetroot & P lacebo & M IV C & $?$ & $?$ & $?$ & + & $?$ & (3) & \\
\hline K u sarav ic etal. 2019 [45] & Cross-over & Tart cherry & P lacebo & D 0 M S & + & $?$ & + & + & $?$ & (3) & \\
\hline \begin{tabular}{|l|l} 
M cLeay etal 2012 [12] \\
\end{tabular} & C ross-over & B lu eberry & P lacebo & M IV C & $?$ & + & + & $?$ & $?$ & (3) & \\
\hline M orehan etal2020 [47] & C ross-over & T art cherry & P lacebo & D $0 M \mathrm{~S}$ & $?$ & + & $?$ & + & $?$ & $\Theta$ & \\
\hline M organ etal. 2018 [14] & Cross-over & Cacao & P lacebo & M IV C & + & + & $?$ & + & $?$ & (3) & \\
\hline Peschek etal. 2014 [26] & Cross-over & Cocoa & P lacebo & M IV C & + & + & + & $?$ & $?$ & $\Theta$ & \\
\hline Trom bold etal. 2011 [10] & Cross-over & Pom egranate & P lacebo & M IV C & + & $?$ & + & + & $?$ & (3) & \\
\hline \begin{tabular}{|l} 
Bell etal. 2015 [39] \\
\end{tabular} & Parallel & T art cherry & P lacebo & M IV C & $?$ & $?$ & $\theta$ & + & $?$ & $\Theta$ & + Low risk \\
\hline Bell etal. 2016 [40] & Parallel & T art cherry & P lacebo & M IV C & $?$ & $?$ & - & + & $?$ & $\odot$ & ? Some concerns \\
\hline Brow n etal. 2018 [41] & Parallel & Tart cherry & P lacebo & M IV C & + & $?$ & + & + & $?$ & (3) & High risk \\
\hline C lifford etal. 2016a [13] & Parallel & B eetroot & P lacebo & M IV C & + & $?$ & + & + & $?$ & (3) & \\
\hline \begin{tabular}{|l|l|} 
C lifford etal. 2016b [27] \\
\end{tabular} & Parallel & B eetroot (high vs. low) & P lacebo & M IV C & $?$ & $?$ & + & + & $?$ & (3) & \\
\hline C lifford etal. 2017a [35] & Parallel & Beetroot & P lacebo & M IV C & $?$ & + & + & + & $?$ & (?) & \\
\hline C lifford etal. 2017b [42] & Parallel & B eetroot & P lacebo & M IV C & + & $?$ & + & + & $?$ & (?) & \\
\hline H ow atson etal. 2010 [36] & Parallel & T art cherry/ap p le blend & P lacebo & M IV C & $?$ & 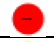 & + & $\odot$ & $?$ & $\odot$ & \\
\hline $\mathrm{H}$ u tch ison etal. 2016 [44] & Parallel & B lack cu rrant nectar & P lacebo & D O M S & $?$ & $?$ & - & + & - & E & \\
\hline K uehletal. 2010 [37] & Parallel & Tart cherry & P lacebo & D 0 M S & + & $?$ & + & + & $?$ & 4 & \\
\hline Lam b etal. 2019 [28] & Parallel & Tart cherry \& pom egranate & P lacebo & M IV C & + & + & + & + & $?$ & (3) & \\
\hline L im a etal. 2019 [46] & Parallel & M ixed fru it $\mathrm{j}$ ice & P lacebo & M IV C & + & $?$ & + & + & $?$ & $\Theta$ & \\
\hline Lynn etal. 2018 [11] & Parallel & B ilberry & P lacebo & D $0 M \mathrm{~S}$ & + & + & + & + & $?$ & (3) & \\
\hline M ach in etal. 2014 [25] & Parallel & Pom egranate (high vs. low) & P lacebo & M IV C & + & $?$ & + & + & $?$ & (3) & \\
\hline $\mathrm{Q} \mathrm{u}$ in lan and H ill 2020 [48] & Parallel & Tart cherry & P lacebo & M IV C & $?$ & $?$ & + & e & $?$ & & \\
\hline
\end{tabular}

Figure 2. Risk of Bias Tool 2 [23]. MIVC: Maximal isometric voluntary contraction; DOMS: Delayed onset muscle soreness.

When the studies were separated by design, the effect sizes were larger in the crossover studies than the parallel studies at all time points, but subgroup analyses only found a statistically significant difference at the $48 \mathrm{~h}$ time-point ( $p=0.02$; see Figure $3 \mathrm{C}$ ).

\section{DOMS}

There was a small but not statistically significant reduction in DOMS immediately post-exercise $(\mathrm{SMD}=-0.23,95 \% \mathrm{CI}-0.53,0.07 ; p=0.14$; participants $=380 ;$ studies $=15$; $\mathrm{I}^{2}=63 \%$; low-quality evidence). At $24 \mathrm{~h}$ post-exercise (SMD $=-0.29,95 \% \mathrm{CI}-0.47,-0.11$; $p=0.002$; participants $=567$; studies $=24 ; \mathrm{I}^{2}=46 \%$; low-quality evidence), $48 \mathrm{~h}$ post-exercise $\left(\mathrm{SMD}=-0.28,95 \% \mathrm{CI}-0.46,-0.09 ; p=0.003 ;\right.$ participants $=567 ;$ studies $=24 ; \mathrm{I}^{2}=44 \%$; 
low-quality evidence) and $72 \mathrm{~h}$ post-exercise (SMD $=-0.46,95 \% \mathrm{CI}-0.69,-0.24 ; p<0.001$; participants $=396$; studies $=16 ; \mathrm{I}^{2}=47 \%$; very low-quality evidence) there were small but statistically significant reductions in DOMS in response to polyphenol supplementation. At $96 \mathrm{~h}$, DOMS had returned towards baseline levels and there was no statistically significant difference between polyphenols and placebo $(\mathrm{SMD}=-0.10,95 \% \mathrm{CI}-0.30,0.10 ; p=0.32$; participants $=158$; studies $=5 ; I^{2}=0 \%$; low-quality evidence) (see Figure 4 ). These SMDs were equivalent to reductions in DOMS of $10.5 \%$ (immediately post), $9.4 \%(24 \mathrm{~h}), 10.3 \%$ (48 h), 29.3\% (72 h) and 9.3\% (96 h). Subgroup analyses did not detect any statistically significant differences in SMDs between cross-over and parallel studies at any post-exercise time point.

\section{CMJ}

Immediately post-exercise $(\mathrm{SMD}=0.27,95 \% \mathrm{CI} 0.01,0.53 ; p=0.04 ;$ participants $=190$; studies $=8 ; \mathrm{I}^{2}=0 \%$; low-quality evidence) and at $24 \mathrm{~h}$ post-exercise ( $\mathrm{SMD}=0.47$, $95 \%$ CI $0.11,0.83 ; p=0.01$; participants $=226$; studies $=10 ; \mathrm{I}^{2}=67 \%$; very low-quality evidence) there were small statistically significant effects of polyphenol supplementation on CMJ height. At $48 \mathrm{~h}(\mathrm{SMD}=0.58,95 \% \mathrm{CI} 0.24,0.91 ; p<0.001$; participants $=248$; studies $=11 ; \mathrm{I}^{2}=69 \%$; very low-quality evidence) and $72 \mathrm{~h}(\mathrm{SMD}=0.57,95 \% \mathrm{CI} 0.03,1.10 ; p=0.04$; participants $=152$; studies $=7 ; \mathrm{I}^{2}=80 \%$; very low-quality evidence) post-exercise there were medium-sized effects of polyphenol supplementation on enhancing recovery of CMJ height (see Figure 5). These SMDs were equivalent to increases in CMJ height of 5.5\% (immediately post), $9.9 \%(24 \mathrm{~h}), 13.1 \%(48 \mathrm{~h}$ ) and $8.0 \%$ (72 h).

When the studies were separated by design, the SMDs were larger in the parallel studies at all time points except immediately post-exercise. However, subgroup analyses only revealed a statistically significant difference at $48 \mathrm{~h}(p=0.02$; see Figure $5 \mathrm{C})$.

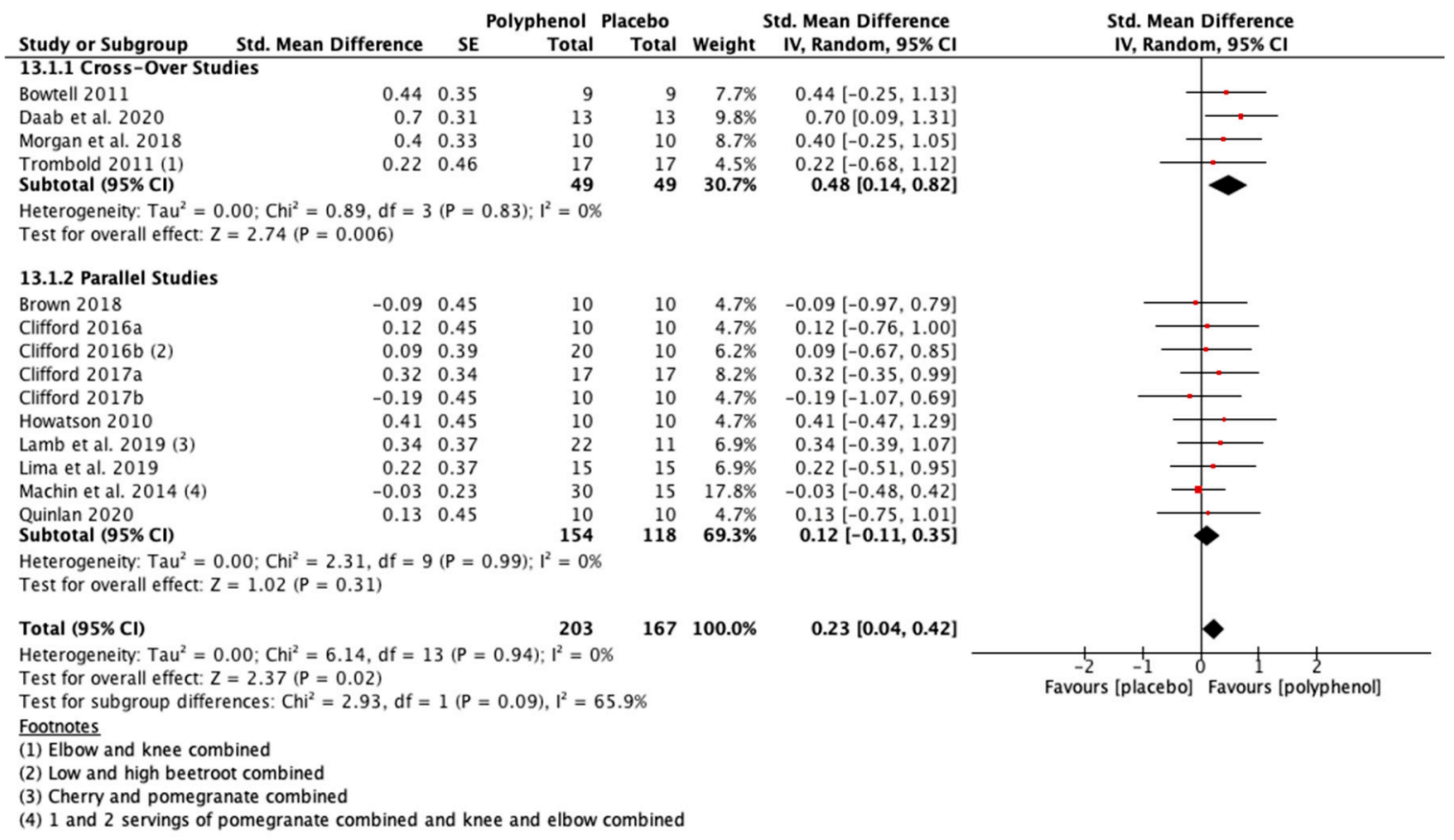




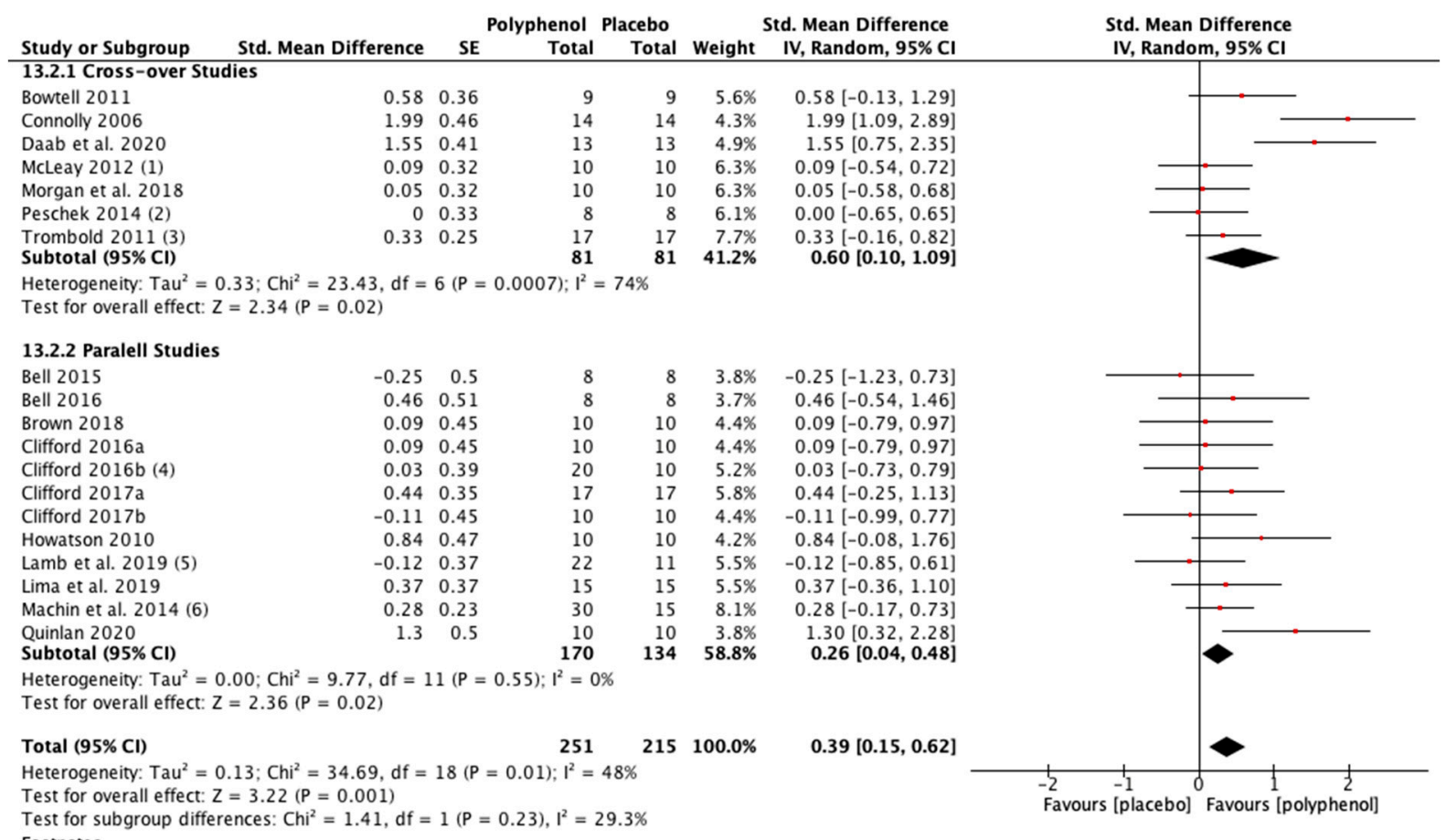

\section{Footnotes}

(2) Left and right leg combined

(3) Elbow and knee combined

(4) Low and high beetroot combined

(5) Cherry and pomegranate combined

(6) 1 and 2 servings of pomegranate combined and knee and elbow combined

(B)

Polyphenol Placebo

\begin{tabular}{lrrrrrr} 
& & \multicolumn{2}{c}{ Polyphenol Placebo } & \multicolumn{2}{c}{ Std. Mean Difference } \\
Study or Subgroup & \multicolumn{1}{c}{ Std. Mean Difference } & SE & Total & Total & Weight & IV, Random, 95\% Cl \\
\hline 13.3.1 Cross-over Studies & & & & & & \\
Bowtell 2011 & 0.42 & 0.35 & 9 & 9 & $5.7 \%$ & $0.42[-0.27,1.11]$ \\
Connolly 2006 & 1.61 & 0.4 & 14 & 14 & $4.7 \%$ & $1.61[0.83,2.39]$ \\
Daab et al. 2020 & 1.06 & 0.35 & 13 & 13 & $5.7 \%$ & $1.06[0.37,1.75]$ \\
McLeay 2012 (1) & 0.58 & 0.34 & 10 & 10 & $5.9 \%$ & $0.58[-0.09,1.25]$ \\
Morgan et al. 2018 & 0.4 & 0.33 & 10 & 10 & $6.1 \%$ & $0.40[-0.25,1.05]$ \\
Peschek 2014 (2) & 0.57 & 0.27 & 8 & 8 & $7.9 \%$ & $0.57[0.04,1.10]$ \\
Trombold 2011) (3) & 0.56 & 0.26 & 17 & 17 & $8.2 \%$ & $0.56[0.05,1.07]$ \\
Subtotal (95\% Cl) & & & $\mathbf{8 1}$ & $\mathbf{8 1}$ & $\mathbf{4 4 . 2 \%}$ & $\mathbf{0 . 7 0}[\mathbf{0 . 4 1} \mathbf{0 . 9 8}$
\end{tabular}

Heterogeneity: $\mathrm{Tau}^{2}=0.04 ; \mathrm{Chi}^{2}=8.32, \mathrm{df}=6(\mathrm{P}=0.22) ; \mathrm{I}^{2}=28 \%$

Test for overall effect: $Z=4.85(P<0.00001)$

13.3.2 Paralell Studies

$\begin{array}{lrrrrrr}\text { Bell 2015 } & 0.13 & 0.5 & 8 & 8 & 3.3 \% & 0.13[-0.85,1.11] \\ \text { Bell 2016 } & 0.49 & 0.51 & 8 & 8 & 3.2 \% & 0.49[-0.51,1.49] \\ \text { Brown 2018 } & 0.24 & 0.45 & 10 & 10 & 3.9 \% & 0.24[-0.64,1.12] \\ \text { Clifford 2016a } & 0.12 & 0.45 & 10 & 10 & 3.9 \% & 0.12[-0.76,1.00] \\ \text { Clifford 2016b (4) } & 0.16 & 0.39 & 20 & 10 & 4.9 \% & 0.16[-0.60,0.92] \\ \text { Clifford 2017a } & 0.23 & 0.34 & 17 & 17 & 5.9 \% & 0.23[-0.44,0.90] \\ \text { Clifford 2017b } & -0.07 & 0.45 & 10 & 10 & 3.9 \% & -0.07[-0.95,0.81] \\ \text { Howatson 2010 } & 0.8 & 0.47 & 10 & 10 & 3.7 \% & 0.80[-0.12,1.72] \\ \text { Lamb et al. 2019(5) } & -0.06 & 0.37 & 22 & 11 & 5.3 \% & -0.06[-0.79,0.67] \\ \text { Lima et al. 2019 } & 0.53 & 0.37 & 15 & 15 & 5.3 \% & 0.53[-0.20,1.26] \\ \text { Machin et al. 2014 (6) } & 0.11 & 0.22 & 30 & 15 & 9.7 \% & 0.11[-0.32,0.54] \\ \text { Quinlan 2020 } & 1.7 & 0.54 & 10 & 10 & 2.9 \% & 1.70[0.64,2.76] \\ \text { Subtotal (95\% CI) } & & & \mathbf{1 7 0} & \mathbf{1 3 4} & \mathbf{5 5 . 8} \% & \mathbf{0 . 2 7}[0.05, \mathbf{0 . 4 9}]\end{array}$

Heterogeneity: $\mathrm{Tau}^{2}=0.00 ; \mathrm{Chi}^{2}=11.15, \mathrm{df}=11(\mathrm{P}=0.43) ; \mathrm{I}^{2}=1 \%$ Test for overall effect: $Z=2.44(P=0.01)$

\section{Total $(95 \% \mathrm{Cl})$}

$251 \quad 215 \quad 100.0 \%$

Heterogeneity: $\mathrm{Tau}^{2}=0.06 ; \mathrm{Chi}^{2}=25.71, \mathrm{df}=18(\mathrm{P}=0.11) ; \mathrm{I}^{2}=30 \%$

Test for overall effect: $Z=4.74$ ( $P<0.00001$ )

Test for subgroup differences: $\mathrm{Chi}^{2}=5.39, \mathrm{df}=1(\mathrm{P}=0.02), \mathrm{I}^{2}=81.5 \%$

$0.48[0.28,0.67]$

Footnotes

(1) $36 \mathrm{~h}$ post

(2) Left and right leg combined

(3) Elbow and knee combined

(4) Low and high beetroot combined

(5) Cherry and pomegranate combined

(6) 1 and 2 servings of pomegranate combined and knee and elbow combined

Figure 3. Cont. 


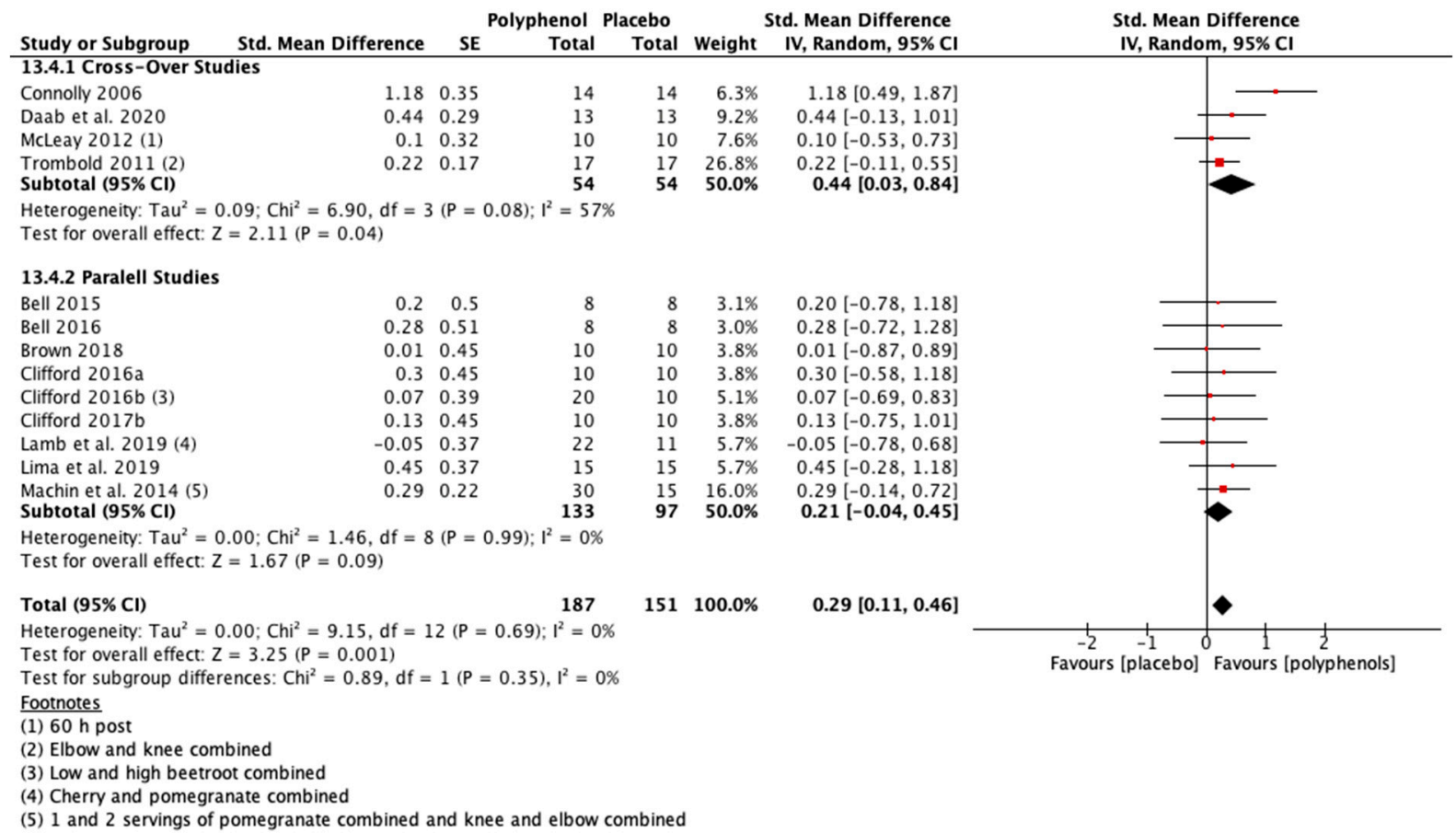

(D)

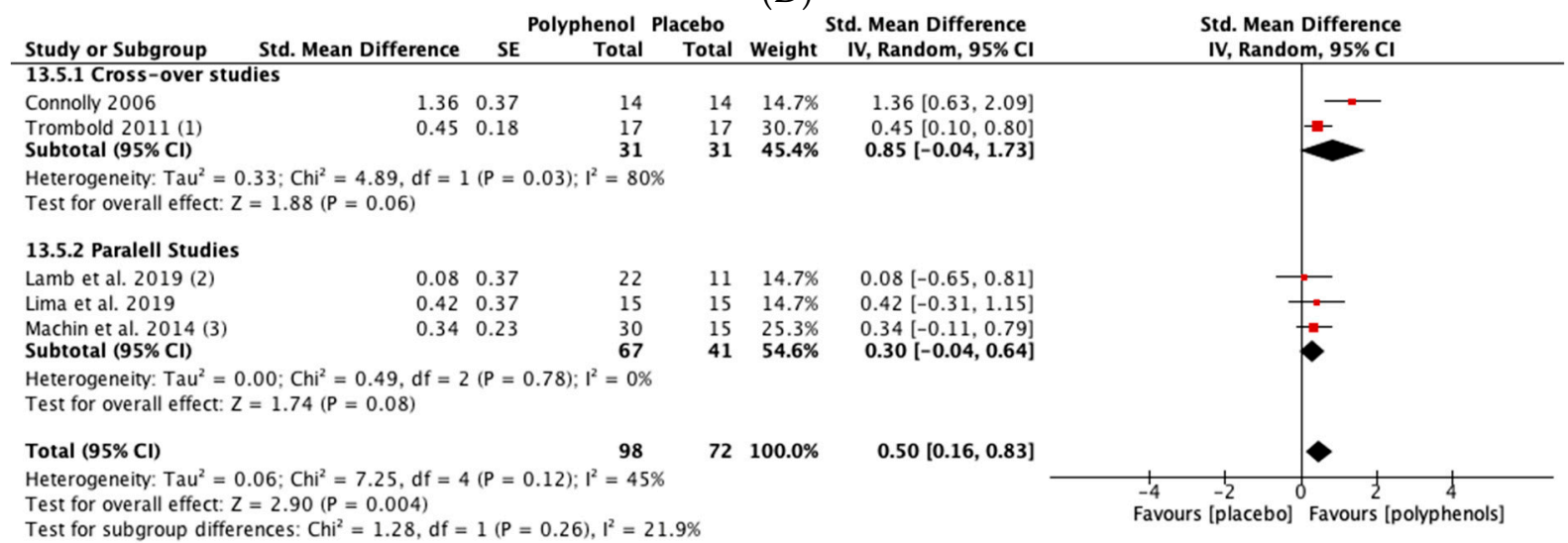

Test for subgroup differences: $\mathrm{Chi}^{2}=1.28, \mathrm{df}=1(\mathrm{P}=0.26), \mathrm{I}^{2}=21.9 \%$

Footnotes

(1) Elbow and knee combined

(2) Cherry and pomegranate combined

(3) 1 and 2 servings of pomegranate combined and knee and elbow combined

(E)

Figure 3. Effect of polyphenol-rich foods, juices and concentrates on recovery of maximal voluntary isometric contraction A) immediately post-exercise; (B) $24 \mathrm{~h}$; (C) $48 \mathrm{~h}$; (D) $72 \mathrm{~h}$; (E) $96 \mathrm{~h}$.

Heterogeneity

We detected substantial heterogeneity at $24 \mathrm{~h}$ post-exercise for MIVC $\left(\mathrm{I}^{2}=48 \%\right.$; $\left.\chi^{2}=34.69, \mathrm{df}=18 ; p=0.01\right)$, immediately $\left(\mathrm{I}^{2}=63 \% ; \chi^{2}=37.74, \mathrm{df}=14 ; p<0.001\right), 24 \mathrm{~h}$ $\left(\mathrm{I}^{2}=46 \% ; \chi^{2}=42.88, \mathrm{df}=23 ; p=0.007\right), 48 \mathrm{~h}\left(\mathrm{I}^{2}=44 \% ; \chi^{2}=41.43, \mathrm{df}=23 ; p=0.01\right)$ and $72 \mathrm{~h}$ post-exercise $\left(\mathrm{I}^{2}=47 \% ; \chi^{2}=28.16, \mathrm{df}=15 ; p=0.02\right)$ for DOMS, and at $24 \mathrm{~h}$ $\left(\mathrm{I}^{2}=67 \% ; \chi^{2}=27.62, \mathrm{df}=9 ; p=0.001\right)$ and $48 \mathrm{~h}\left(\mathrm{I}^{2}=69 \% ; \chi^{2}=31.79, \mathrm{df}=10 ; p<0.001\right)$ post-exercise for $\mathrm{CMJ}$ height. Exploration of heterogeneity revealed that different studies were driving the substantial heterogeneity for each primary outcome. Exclusion of these studies removed the heterogeneity and attenuated the SMDs for DOMS and MIVC, but treatment effects remained statistically significant. For CMJ, removing studies causing substantial heterogeneity had almost no effect on the SMDs. 


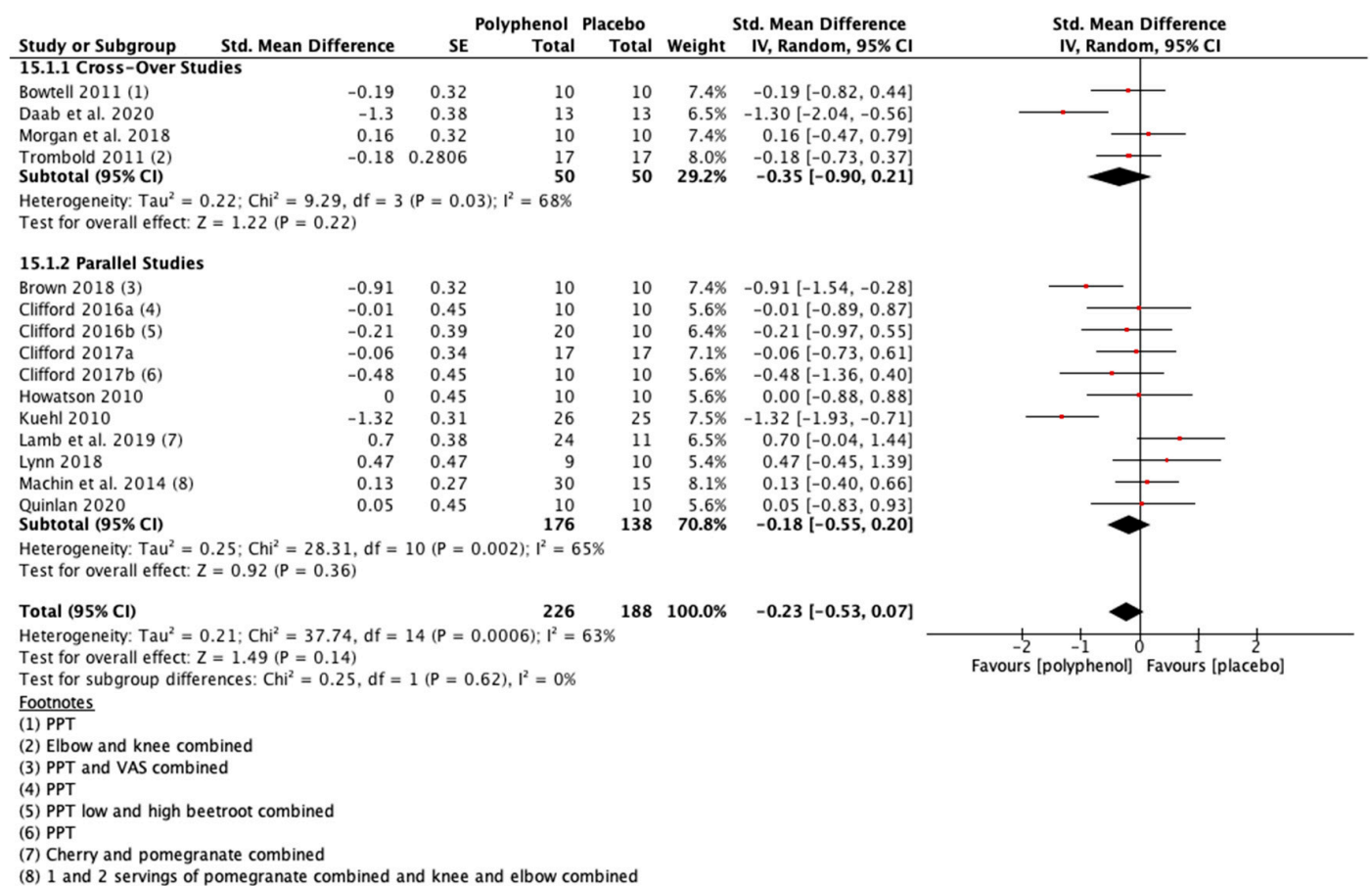

(A)

Polyphenol Placebo Std. Mean Difference Std. Mean Difference
IV, Random, $95 \% \mathrm{CI}$

Study or Subgroup

Std. Mean Difference SE Polyphenol Place Std. Mean Difference

$\begin{array}{lrrrrrr}\text { Abbot et al. 2019 (1) } & 0.11 & 0.34 & 10 & 10 & 4.2 \% & 0.11[-0.56,0.78] \\ \text { Bowtell 2011 (2) } & -0.28 & 0.34 & 10 & 10 & 4.2 \% & -0.28[-0.95,0.39] \\ \text { Connolly 2006 (3) } & -0.15 & 0.19 & 14 & 14 & 7.0 \% & -0.15[-0.52,0.22] \\ \text { Daab et al. 2020 } & -1.46 & 0.4 & 13 & 13 & 3.5 \% & -1.46[-2.24,-0.68] \\ \text { Kupusaravic et al. 2019 } & -0.45 & 0.33 & 10 & 10 & 4.4 \% & -0.45[-1.10,0.20] \\ \text { McLeay 2012 (4) } & -0.17 & 0.32 & 10 & 10 & 4.5 \% & -0.17[-0.80,0.46] \\ \text { Morehen 2020 } & 0 & 0.3 & 11 & 11 & 4.8 \% & 0.00[-0.59,0.59] \\ \text { Morgan et al. 2018 } & 0.15 & 0.32 & 10 & 10 & 4.5 \% & 0.15[-0.48,0.78] \\ \text { Peschek 2014 (5) } & -0.53 & 0.22 & 8 & 8 & 6.3 \% & -0.53[-0.96,-0.10] \\ \text { Trombold 2011 (6) } & -0.25 & 0.18 & 17 & 17 & 7.2 \% & -0.25[-0.60,0.10] \\ \text { Subtotal (95\% CI) } & & & \mathbf{1 1 3} & \mathbf{1 1 3} & \mathbf{5 0 . 7 \%} & -0.27[-0.49,-0.05]\end{array}$
Srombold 2011 (6) $d f=9(P=0.10) ; I^{2}=39 \%$ $\begin{array}{rrr}113 & 7.2 \% & -0.25[-0.60,0.10\end{array}$ Heterogeneity: $\mathrm{Tau}^{2}=0.05 ; \mathrm{Chi}^{2}=14.83$, d
Test for overall effect: $\mathrm{Z}=2.43(\mathrm{P}=0.02$

15.2.2 Parallel studies

Bell 2015

Bell 2016

Brown 2018 (7)

Clifford 2016a (8)

Clifford 2016b (9)

Clifford 2017a

Clifford 2017b (10)

Howatson 2010

Hutchison 2016
Lamb et al. 2019 (11)

Lima et al. 2019

Lynn 2018

Machin et al. 2014 (12)

Quinlan 2020

Subtotal $(95 \% \mathrm{Cl})$
Heterogeneity: $\mathrm{Tau}^{2}=0.16 ; \mathrm{Chi}^{2}=27.90, \mathrm{df}=13(\mathrm{P}=0.009) ; \mathrm{I}^{2}=53 \%$
Test for overall effect: $\mathrm{Z}=1.99(\mathrm{P}=0.05)$

Total $(95 \% \mathrm{Cl})$

$\begin{array}{ll}-0.17 & 0.5 \\ -0.73 & 0.52\end{array}$

$\begin{array}{lll}-0.73 & 0.52\end{array}$

$\begin{array}{lll}-1.09 & 0.24\end{array}$

$-0.090 .45$

$\begin{array}{ll}-1.08 & 0.42 \\ -0.04 & 0.34\end{array}$

$\begin{array}{ll}-0.04 & 0.34 \\ -0.47 & 0.45\end{array}$

$\begin{array}{rr}-0.47 & 0.45 \\ 0.2 & 0.45\end{array}$

$\begin{array}{cc}-0.3 & 0.51 \\ 0.61 & 0.37\end{array}$

$\begin{array}{ll}0.61 & 0.37\end{array}$

$\begin{array}{rr}-0.64 & 0.38 \\ 0.02 & 0.46 \\ 0.09 & 0.22\end{array}$

$\begin{array}{rr}0.02 & 0.46 \\ 0.09 & 0.22\end{array}$

$-0.520 .46$

$\begin{array}{llll}8 & 8 & 2.5 \% & -0.17[-1.15,0.81] \\ 8 & 8 & 2.4 \% & -0.73[-1.75,0.29]\end{array}$

$\begin{array}{rrrr}8 & 8 & 2.4 \% & -0.73[-1.75,0.29] \\ 10 & 10 & 5.9 \% & -1.09[-1.56,-0.62]\end{array}$

$10 \quad 10 \quad 3.0 \% \quad-0.09[-0.97,0.79]$

$\begin{array}{llll}20 & 10 & 3.3 \% & -1.08[-1.90,-0.26\end{array}$

$4.2 \%-0.04[-0.71,0.63]$

$3.0 \%-0.47[-1.35,0.41]$

$3.0 \% \quad 0.20[-0.68,1.08]$

$2.5 \%-0.30[-1.30,0.70]$

$3.8 \% \quad 0.61[-0.12,1.34]$

$3.7 \%-0.64[-1.38,0.10]$

$2.9 \% \quad 0.02[-0.88,0.92]$

$6.3 \% \quad 0.09[-0.34,0.52]$ $\begin{array}{rrr}10 & 2.9 \% & -0.52[-1.42,0.38] \\ 152 & 49.3 \% & -0.30[-0.60,-0.00]\end{array}$

$265 \quad 100.0 \%-0.29[-0.47,-0.11]$

Heterogeneity: $\mathrm{Tau}^{2}=0.09 ; \mathrm{Chi}^{2}=42.88, \mathrm{df}=23(\mathrm{P}=0.007): \mathrm{I}^{2}=46 \%$

Test for overall effect: $Z=3.14(P=0.002)$

Test for subgroup differences: $\mathrm{Chi}^{2}=0.02, \mathrm{df}=1(\mathrm{P}=0.87), \mathrm{I}^{2}=0 \%$

Footnotes

(1) $12 \mathrm{~h}$ post

(2) $P P$

3) PPT and VAS combine

(4) $12 \mathrm{~h}$ post

(5) PPT and VAS combined

(6) Elbow and knee combined

(7) PPT and VAS combined

(8) PPT

9) PPT low and high beetroot combined

(10) PPT

(11) Cherry and pomegranate combine

(12) 1 and 2 servings of pomegranate combined and knee and elbow combined

(B)

Figure 4. Cont. 


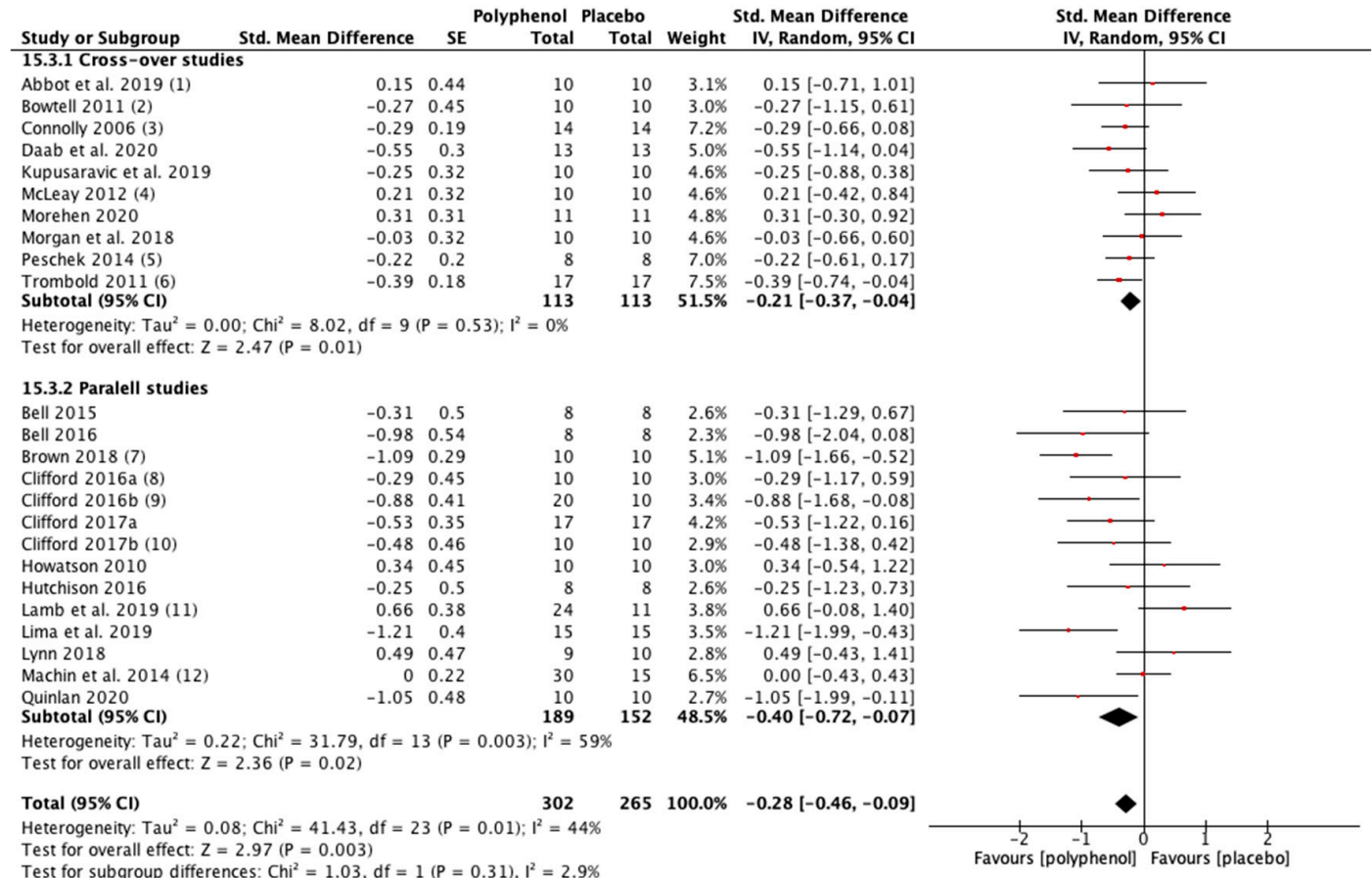

Test for subgroup differences: $\mathrm{Ch}^{2}=1.03, \mathrm{df}=1(\mathrm{P}=0.31), \mathrm{I}^{2}=2.9 \%$

Favours [polyphenol] Favours [placebo]

Footnotes

(1) $36 \mathrm{~h}$ post

(2) PPT

(3) PPT and VAS combined

(4) $36 \mathrm{~h}$ post

(5) PPT and VAS combined

(7) Elbow and knee combined

(7) PPT

(9) PPT low and high beetroot combined

(10) PPT

(11) Cherry and pomegranate combined

(12) 1 and 2 servings of pomegranate combined and knee and elbow combined

(C)

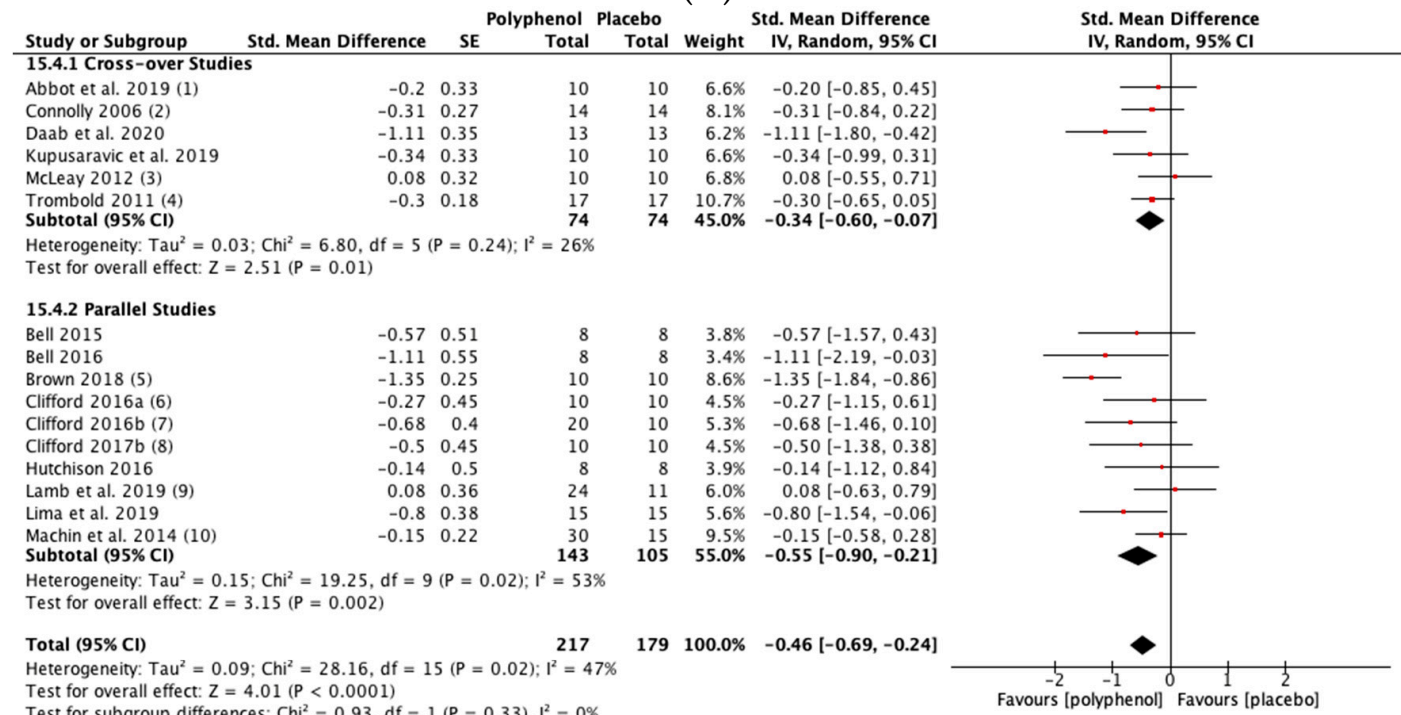

Test for subgroup differences: $\mathrm{Chi}^{2}=0.93, \mathrm{df}=1(\mathrm{P}=0.33), \mathrm{I}^{2}=0 \%$

Footnotes

(1) $60 \mathrm{~h}$ post

(2) PPT and VAS combined

(3) $60 \mathrm{~h}$ post

(4) Elbow and knee combined

(5) PPT and VAS combined

(7) PPT low and high beetroot combined

(8) PPT

(9) Cherry and pomegranate combined

(10) 1 and 2 servings of pomegranate combined and knee and elbow combined

Figure 4. Cont. 


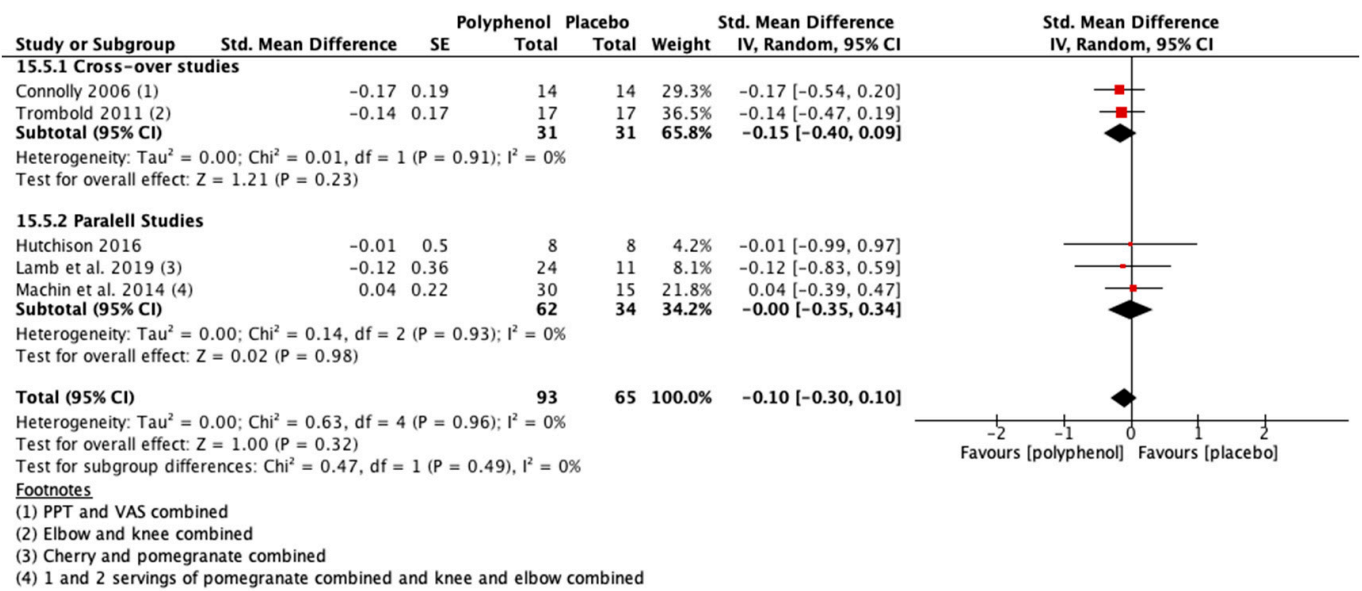

(E)

Figure 4. Effect of polyphenol-rich foods, juices and concentrates on recovery of delayed onset muscle soreness (A) immediately post-exercise; (B) 24 h; (C) 48 h; (D) 72 h; (E) 96 h. Unless stated otherwise SMDs are calculated from VAS of DOMS. PPT: Pain pressure threshold. VAS: Visual analogue scale.

Sensitivity Analysis

A sensitivity analysis excluding 10 studies classified as high risk of bias (see Supplementary File 4) attenuated the SMDs for MIVC, but the effects of polyphenol supplementation remained statistically significant. Removal of the high risk studies had little effect on the SMDs for DOMS. The SMDs for CMJ height were attenuated and no longer statistically significant immediately post-exercise (SMD $=0.25,95 \% \mathrm{CI}-0.03,0.53 ; p=0.08$ ) and at the $24(\mathrm{SMD}=0.25 .95 \% \mathrm{CI}-0.03,0.53 ; p=0.08)$ and $72 \mathrm{~h}$ time-points $(\mathrm{SMD}=0.44$, $95 \%$ CI $-0.09,0.97 ; p=0.10$ ) (see Supplementary File 4).

\subsubsection{Secondary Outcomes}

CK

There were no statistically significant effects of polyphenol supplementation on CK immediately post-exercise $(\mathrm{SMD}=-0.05,95 \% \mathrm{CI}-0.35,0.25 ; p=0.75$; participants $=315$; studies $\left.=14 ; \mathrm{I}^{2}=52 \%\right), 24 \mathrm{~h}$ post-exercise $(\mathrm{SMD}=0.11,95 \% \mathrm{CI}-0.10,0.32 ; p=0.30$; participants $=367 ;$ studies $\left.=17 ; \mathrm{I}^{2}=27 \%\right), 48 \mathrm{~h}$ post-exercise $(\mathrm{SMD}=0.07,95 \% \mathrm{CI}-0.19,0.33$; $p=0.62$; participants $=397 ;$ studies $\left.=18 ; \mathrm{I}^{2}=55 \%\right)$ and $72 \mathrm{~h}$ post-exercise $(\mathrm{SMD}=-0.14,95 \%$ CI $-0.52,0.24 ; p=0.47 ;$ participants $=202 ;$ studies $=9 ; \mathrm{I}^{2}=52 \%$ ). Only three parallel studies measured CK at $96 \mathrm{~h}$ post-exercise; these revealed a moderate-sized effect in favour of polyphenol supplementation $(\mathrm{SMD}=-0.62,95 \% \mathrm{CI}-1.25,0.00 ; p=0.05$; participants $=80$; studies $=3 ; I^{2}=41 \%$ ) (see Supplementary File 5).

\section{CRP}

There were no statistically significant effects of polyphenol supplementation on CRP immediately post-exercise $(\mathrm{SMD}=0.01,95 \% \mathrm{CI}-0.33,0.34 ; p=0.97$; participants $=229$; studies $\left.=11 ; \mathrm{I}^{2}=45 \%\right), 24 \mathrm{~h}(\mathrm{SMD}=0.00,95 \% \mathrm{CI}-0.37,0.38 ; p=0.98 ;$ participants $=249 ;$ studies $\left.=12 ; \mathrm{I}^{2}=60 \%\right), 48 \mathrm{~h}(\mathrm{SMD}=0.04,95 \% \mathrm{CI}-0.32,0.41 ; p=0.81 ;$ participants $=249$; studies $\left.=12 ; \mathrm{I}^{2}=56 \%\right)$ and $72 \mathrm{~h}$ post-exercise $(\mathrm{SMD}=-0.12,95 \% \mathrm{CI}-0.50,0.26 ; p=0.54$; participants $=118$; studies $=6 ; \mathrm{I}^{2}=18 \%$ ). No studies measured CRP at $96 \mathrm{~h}$ post-exercise (see Supplementary File 6).

\section{IL-6}

Polyphenol supplementation had no statistically significant effect on IL-6 immediately $\left(\mathrm{SMD}=-0.34,95 \% \mathrm{CI}-0.76,0.08 ; p=0.11 ;\right.$ participants $=156 ;$ studies $\left.=7 ; \mathrm{I}^{2}=57 \%\right)$, $24 \mathrm{~h}\left(\mathrm{SMD}=-0.18,95 \% \mathrm{CI}-0.64,0.28 ; p=0.44 ;\right.$ participants $=150 ;$ studies $\left.=7 ; \mathrm{I}^{2}=56 \%\right)$, 
$48 \mathrm{~h}\left(\mathrm{SMD}=-0.19,95 \% \mathrm{CI}-0.68,0.30 ; p=0.45 ;\right.$ participants $=172 ;$ studies $\left.=8 ; \mathrm{I}^{2}=78 \%\right)$, and $72 \mathrm{~h}$ post-exercise $(\mathrm{SMD}=-0.56,95 \% \mathrm{CI}-1.25,-0.13 ; p=0.11$; participants $=82$; studies $=4 ; \mathrm{I}^{2}=61 \%$ ). Only one study measured IL-6 at $96 \mathrm{~h}$ post-exercise which showed a moderate but not statistically significant effect $(\mathrm{SMD}=-0.76,95 \% \mathrm{CI}-1.80,0.28 ; p=0.15$; participants $=16$; studies $=1)($ see Supplementary File 7$)$.

PC

Immediately post-exercise $(\mathrm{SMD}=0.46,95 \% \mathrm{CI}-0.67,1.60 ; p=0.42 ;$ participants $=76$; studies $\left.=4 ; \mathrm{I}^{2}=82 \%\right)$, at $24 \mathrm{~h}(\mathrm{SMD}=0.39,95 \% \mathrm{CI}-0.24,1.01 ; p=0.22 ;$ participants $=96$; studies $=5 ; \mathrm{I}^{2}=66 \%$ ) and $48 \mathrm{~h}$ post-exercise $(\mathrm{SMD}=0.33,95 \% \mathrm{CI}-0.25,0.92 ; p=0.27$; participants $=96$; studies $=5 ; \mathrm{I}^{2}=63 \%$ ) polyphenol supplementation caused small increases in PC relative to placebo, although the differences were not statistically significant. There were only two studies (one parallel and one cross-over) that measured PC at $72 \mathrm{~h}$ postexercise; these indicated a moderate-sized effect in favour of polyphenol supplementation $\left(\mathrm{SMD}=-0.64,95 \% \mathrm{CI}-1.14,-0.14 ; p=0.01 ;\right.$ participants $=40 ;$ studies $\left.=2 ; \mathrm{I}^{2}=0 \%\right)$. No studies measured PC at 96 h post-exercise (see Supplementary File 8).

\section{$\begin{array}{cc}\text { Std. Mean Difference } & \text { Std. Mean Difference } \\ \text { IV, Random, } 95 \% \mathrm{CI}\end{array}$} IV, Random, 95\% CI

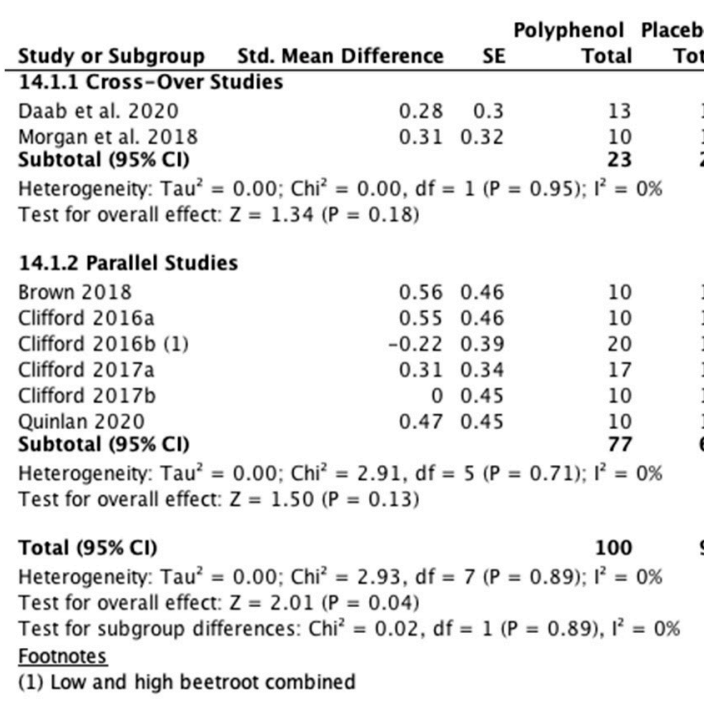

\begin{tabular}{|c|c|c|c|}
\hline \multirow{2}{*}{ Study or Subgroup } & \multirow[b]{2}{*}{ Std. Mean Difference } & \multicolumn{2}{|c|}{ Polyphenol Placeb } \\
\hline & & SE & Total \\
\hline \multicolumn{4}{|c|}{ 14.1.1 Cross-Over Studies } \\
\hline Daab et al. 2020 & 0.28 & 0.3 & 13 \\
\hline Morgan et al. 2018 & 0.31 & 0.32 & 10 \\
\hline \multirow{2}{*}{\multicolumn{4}{|c|}{$\begin{array}{l}\text { Heterogeneity: } \mathrm{Tau}^{2}=0.00 ; \mathrm{Chi}^{2}=0.00, \mathrm{df}=1(\mathrm{P}=0.95) ; \mathrm{I}^{2}=0 \% \\
\text { Test for overall effect: } \mathrm{Z}=1.34(\mathrm{P}=0.18)\end{array}$}} \\
\hline & & & \\
\hline \multicolumn{4}{|l|}{ 14.1.2 Parallel Studies } \\
\hline Brown 2018 & 0.56 & 0.46 & 10 \\
\hline Clifford 2016a & 0.55 & 0.46 & 10 \\
\hline Clifford 2016b (1) & -0.22 & 0.39 & 20 \\
\hline Clifford 2017a & 0.31 & 0.34 & 17 \\
\hline Clifford 2017b & 0 & 0.45 & 10 \\
\hline Quinlan 2020 & 0.47 & 0.45 & 10 \\
\hline \multirow{2}{*}{\multicolumn{4}{|c|}{$\begin{array}{l}\text { Heterogeneity: } \mathrm{Tau}^{2}=0.00 ; \mathrm{Chi}^{2}=2.91, \mathrm{df}=5(\mathrm{P}=0.71) ; \mathrm{I}^{2}=0 \% \\
\text { Test for overall effect: } \mathrm{Z}=1.50(\mathrm{P}=0.13)\end{array}$}} \\
\hline & & & \\
\hline \multirow{4}{*}{\multicolumn{4}{|c|}{$\begin{array}{l}\text { Total }(95 \% \mathrm{Cl}) \\
\text { Heterogeneity: } \mathrm{Tau}^{2}=0.00 ; \mathrm{Ch}^{2}=2.93, \mathrm{df}=7(\mathrm{P}=0.89) ; \mathrm{I}^{2}=0 \% \\
\text { Test for overall effect: } \mathrm{Z}=2.01(\mathrm{P}=0.04) \\
\text { Test for subgroup differences: } \mathrm{Chi}^{2}=0.02, \mathrm{df}=1(\mathrm{P}=0.89), \mathrm{I}^{2}=0 \%\end{array}$}} \\
\hline & & & \\
\hline & & & \\
\hline & & & \\
\hline \multicolumn{4}{|l|}{ Footnotes } \\
\hline
\end{tabular}

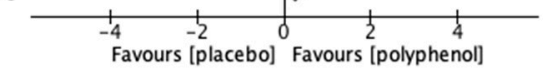

(A)

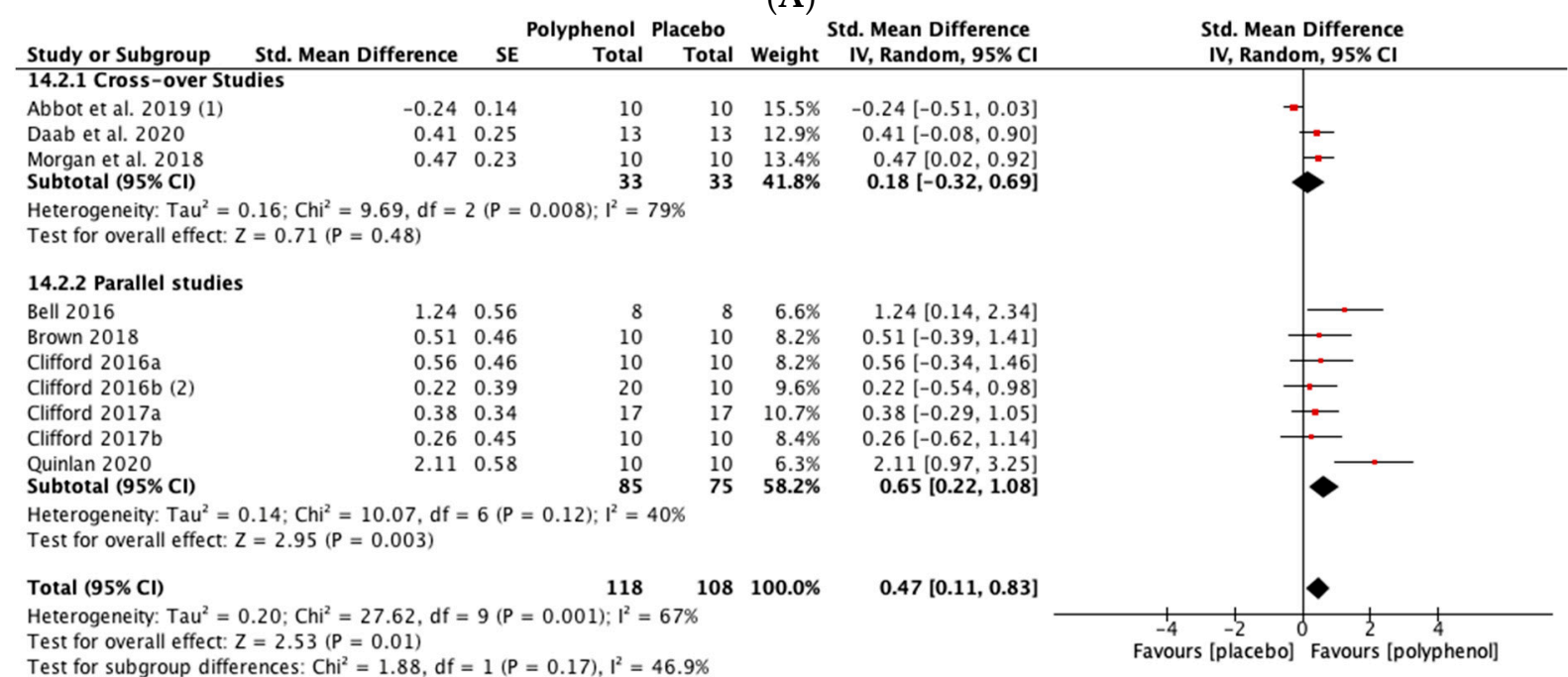




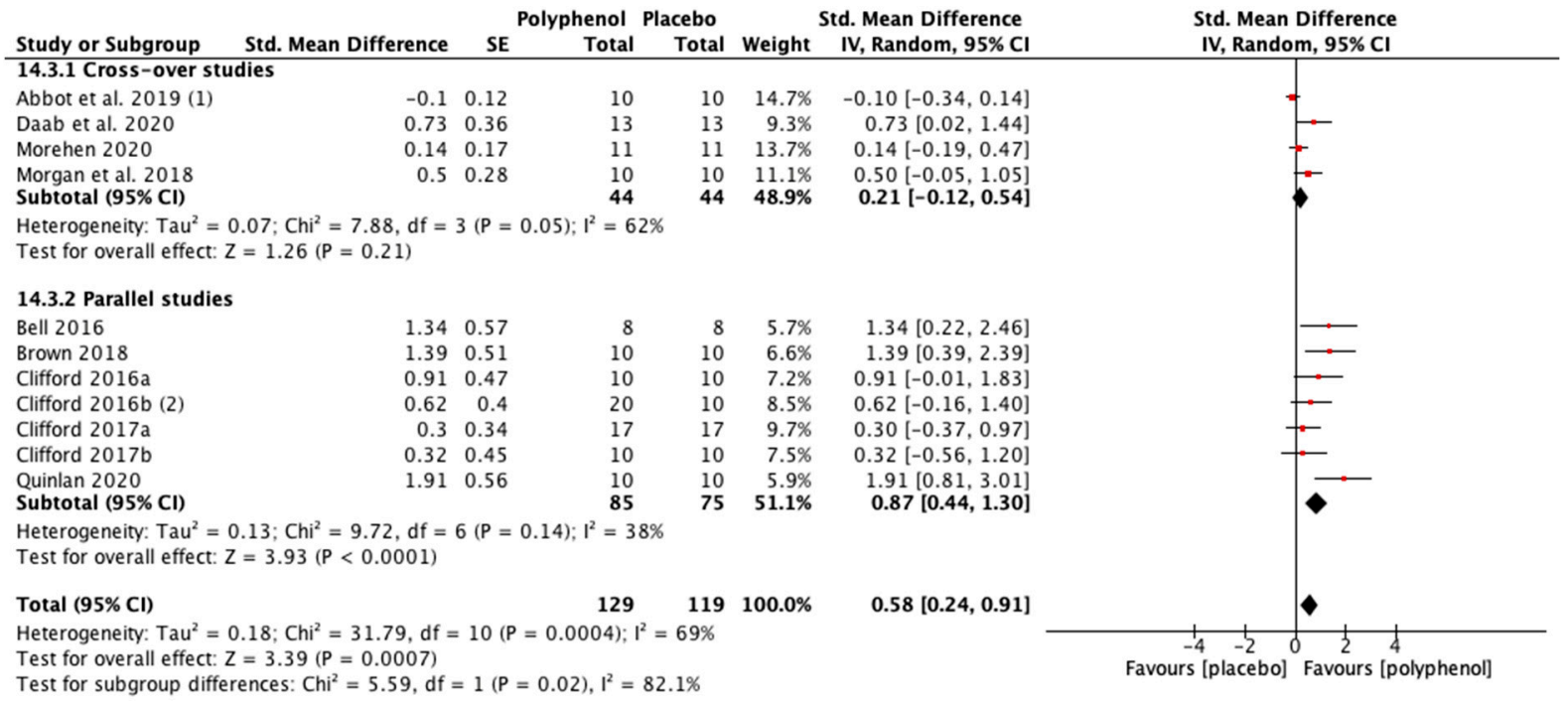

Footnotes

(1) $36 \mathrm{~h}$ post

(2) Low and high beetroot combined

(C)

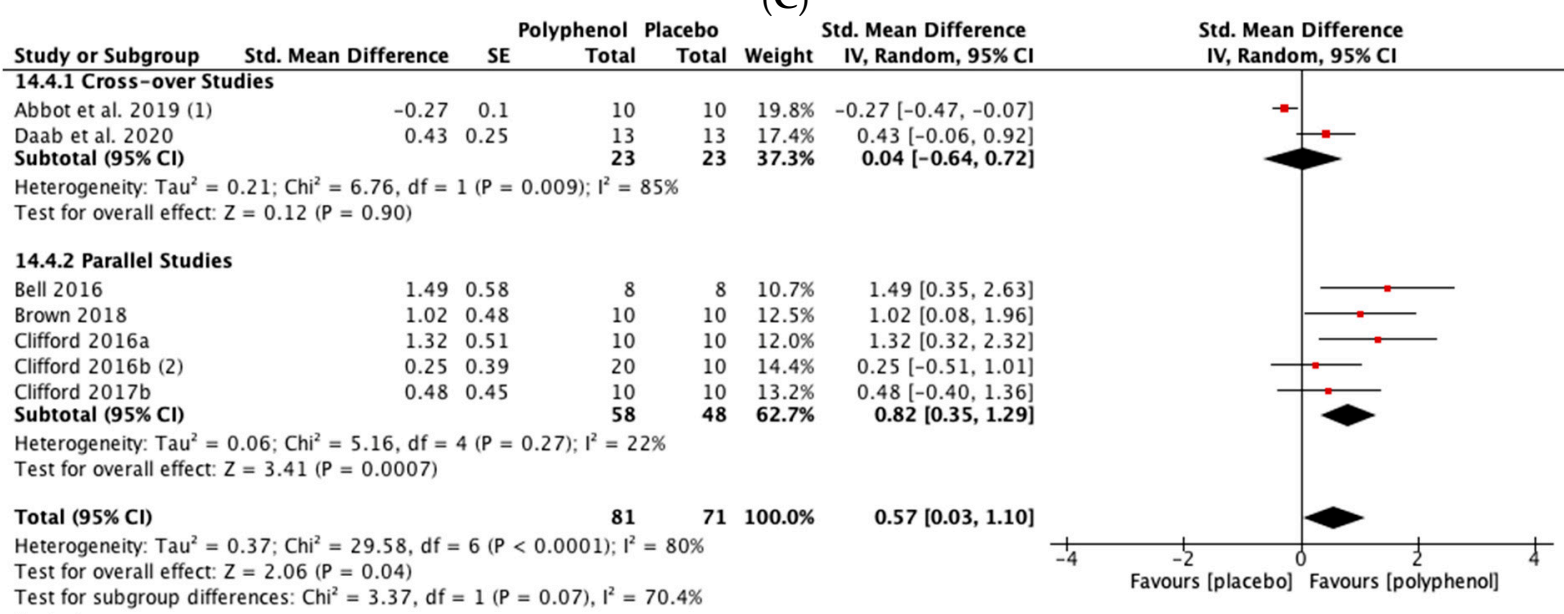

Footnotes

(2) Low and high beetroot combined

(D)

Figure 5. Effect of polyphenol-rich foods, juices and concentrates on recovery of countermovement jump height (A) immediately post-exercise; (B) $24 \mathrm{~h}$; (C) $48 \mathrm{~h}$; (D) $72 \mathrm{~h}$.

\subsubsection{Analyses by Individual Polyphenol-Rich Foods}

Individual meta-analyses and subgroup analyses were conducted for tart cherry $(n=12)$, beetroot $(n=6)$, pomegranate $(n=3)$ and cocoa $(n=2)$. When the studies on tart cherry were analysed alone, the magnitude of the SMDs were greater than for all studies combined for MIVC and CMJ, however, statistical significance was attenuated at most time-points. The effect of tart cherry on DOMS was less than for all studies combined except for $72 \mathrm{~h}$ post-exercise. For pomegranate, the SMDs for MIVC were smaller than for all studies combined and only statistically significant at $96 \mathrm{~h}$ post-exercise. Pomegranate did not cause a statistically significant reduction in DOMS at any of the post-exercise time-points. None of the pomegranate studies measured CMJ height. When individual meta-analyses were conducted for beetroot, the SMDs for DOMS were greater than for all of the studies combined whereas the SMDs for CMJ were slightly attenuated. There were no statistically significant effects of beetroot on MIVC. Two studies analysed the effects of cocoa on recovery of MIVC and DOMS up to $48 \mathrm{~h}$ post-exercise. Analysis revealed a 
statistically significant improvement in MIVC at $48 \mathrm{~h}$ post-exercise but no other statistically significant effects (see Supplementary File 9 for all of the individual analyses).

Subgroup analyses revealed no statistically significant differences for MIVC between pomegranate, cherry, beetroot, and cocoa. There were no statistically significant differences between cherry and beetroot for CMJ. No pomegranate studies and only one cocoa study measured CMJ. Both beetroot and cherry reduced DOMS and there were no statistically significant differences between them at any of the post-exercise time-points. Beetroot caused a statistically significant greater reduction in DOMS than pomegranate at $24(p=0.05)$ and $72(p=0.05) \mathrm{h}$ post-exercise. Cherry caused a greater reduction in DOMS than pomegranate, but the difference was only statistically significant at $72 \mathrm{~h}$ post-exercise $(p=0.05)$. There were no statistically significant differences for DOMS between cocoa and the three other polyphenol supplements (see Supplementary File 9, Table S4).

\subsubsection{Comparison of Studies Based on Restriction of Dietary Polyphenol Intake}

Six studies $[12,27,28,39,40,44]$ directed participants to restrict their intake of dietary polyphenols for the duration of the study, but one reported poor compliance from participants [28]. Comparison of the remaining five studies that restricted polyphenols in the background diet with those studies that did not restrict polyphenols revealed no statistically significant differences for all of the primary outcomes (MIVC, DOMS, and CMJ; see Supplementary File 10 for analyses).

\subsubsection{Quality of Evidence}

The evidence for MIVC was rated moderate to very low across the post-exercise time-points. The evidence was downgraded for serious risk of bias (all time-points), serious inconsistency ( $24 \mathrm{~h}$ and $96 \mathrm{~h}$ ), and serious imprecision (immediately, $72 \mathrm{~h}$ and $96 \mathrm{~h}$ ). For DOMS the evidence was rated low to very low. The evidence was downgraded for serious risk of bias (all time-points), serious inconsistency (immediately, $24 \mathrm{~h}, 48 \mathrm{~h}$ and $72 \mathrm{~h}$ ), and serious imprecision (72 h and $96 \mathrm{~h}$ ). The evidence was rated low to very low for CMJ. The quality of the evidence was downgraded for serious risk of bias (all timepoints), serious inconsistency ( $24 \mathrm{~h}$ and $72 \mathrm{~h}$ ) and serious imprecision (all time-points) (See Supplementary File 3).

\section{Discussion}

In this meta-analysis, polyphenol-rich foods accelerated the recovery of muscle function and reduced muscle soreness post EIMD, however, the certainty of the evidence was moderate to very low. The SMDs for the effects of polyphenol supplementation were small to moderate for our primary outcomes. When the SMDs were converted into percentages, benefits ranged from $5.7 \%$ to $13.0 \%$ for MIVC, $9.3 \%$ to $29.3 \%$ for muscle soreness, and $5.5 \%$ to $13.1 \%$ for $\mathrm{CMJ}$.

A reduction of $14 \%$ [49] has been proposed as the minimal important difference (MID) [50] in muscle soreness. In this study, the difference between polyphenol supplementation and placebo was comparable to the lower threshold of the MID at most post-exercise time-points but much greater at $72 \mathrm{~h}$. Recovery of muscle strength and explosive lower body power after EIMD was commonly assessed by measuring MIVC and CMJ, respectively. To our knowledge, there are no consensus guidelines as to the MIDs for these measures. We found that polyphenol supplementation accelerated recovery of CMJ by between $5.5-13.1 \%$ and MIVC by $5.7-13.0 \%$ in comparison to a placebo. For sports that require explosive lower body power and maximal strength where limited recovery time is available between events, differences of this magnitude could translate into meaningful performance effects, however, there is a need to determine the MID for MIVC and CMJ to aid the interpretation of meta-analyses.

The studies included in this meta-analysis investigated the effect of eight different types of polyphenol-rich plant-based foods. Different plants vary in their profile of polyphenols [51] and other bioactive compounds (e.g., beetroot is rich in nitrate [15] and be- 
talains [16], which could determine their ability to accelerate recovery from EIMD. Thus, we explored the efficacy of individual polyphenol-rich foods. Our meta-analysis of 12 studies on tart cherry found evidence of enhanced recovery of MIVC, CMJ height and muscle soreness at some post-exercise time-points. Analysis of five studies on beetroot found no evidence of a benefit for MIVC, but faster recovery of CMJ and muscle soreness. Whereas, for pomegranate, analysis of three studies found limited evidence of faster recovery of MIVC, but no effect on muscle soreness. For cocoa there was no evidence of a benefit for muscle soreness but there was a faster recovery of MIVC at $48 \mathrm{~h}$ post-exercise. There were insufficient studies to explore the individual effects of the other polyphenol-rich supplements. Subgroup analyses failed to resolve any statistically significant differences between food type for MIVC or CMJ. The reduction in DOMS from beetroot juice was statistically greater than pomegranate juice at $24 \mathrm{~h}$ and $72 \mathrm{~h}$, whereas for tart cherry DOMS was only significantly lower than pomegranate at $72 \mathrm{~h}$. Interpretation of these subgroup analyses needs to be cautious because of the small number of studies included, especially for pomegranate and cocoa.

The ability of polyphenol supplements to accelerate recovery would be expected to be greater in participants that restricted their intake of dietary polyphenols. However, subgroup analyses failed to detect any statistically significant differences in markers of recovery when we compared studies that instructed participants to restrict their diet with those that had no restrictions. A number of limitations in the literature may explain these findings. First, there was substantial heterogeneity in study design, second, there were a limited number of studies $[12,27,39,40,44]$ that restricted polyphenols, third, only two $[27,40]$ of the five studies included a washout period that excluded polyphenol consumption before the intervention, and finally, no studies reported the habitual intakes of dietary polyphenols in their participants, which could have influenced the efficacy of the interventions. Thus, there is a need for future studies in this area to better characterise the polyphenol intakes of their participants.

\subsection{Mechanisms}

Polyphenol supplementation failed to reduce CK at any of the post-exercise timepoints. Serum CK levels are commonly used to assess muscle damage post-exercise, however, the increase in serum CK does not directly correlate with the degree of muscle damage and the loss of force production [18,52]. Also there is substantial inter-individual variability in CK response after muscle damage [53]. Therefore, CK may have limited ability to detect differences in the extent of muscle damage in response to a dietary/recovery intervention $[11,28]$ which probably explains the lack of treatment effect observed in this meta-analysis.

It has been suggested that polyphenols may accelerate recovery through enhancing antioxidant status and supressing inflammation [1]. A number of studies in this review measured total antioxidant status (TAS) in the blood [12,34,36]. We did not conduct a meta-analysis on TAS because it is no longer recommended as a valid method of measuring antioxidant status in vivo [54]. Some studies in this review measured blood-borne markers of oxidative stress such as F2-isoprostanes, lipid hydroperoxides, and PC. We did not pool all of the oxidative stress markers into a single meta-analysis because they are not directly comparable $[55,56]$. We only analysed the data for PC because it was the only oxidative stress marker measured in multiple studies. There was no effect, except at the $72 \mathrm{~h}$ time-point, however, the analysis at $72 \mathrm{~h}$ only included two studies [12,13].

The most commonly analysed markers of inflammation in the studies included in this review were CRP and IL-6. We found no effect of polyphenol supplementation on CRP or IL-6 at any post-exercise time-point. This may be because serum markers do not accurately mirror intramuscular processes [57]. To elucidate the mechanisms through which polyphenols accelerate recovery, future studies should complement serum markers with intramuscular measures of antioxidative status, oxidative stress, and inflammation. 


\subsection{Comparison with Other Reviews}

Our study expands the findings from a previous meta-analysis of fruit supplements and recovery from EIMD by Doma et al. [17]. In agreement with Doma et al. [17] we found that polyphenol supplementation caused a faster recovery of MIVC at $24 \mathrm{~h}$ and $48 \mathrm{~h}$ postexercise but we found smaller SMDs. Notably, Doma et al. (2020) investigated effects only at $24 \mathrm{~h}$ and $48 \mathrm{~h}$ post-exercise despite the recovery process often extending beyond this [2]. Whereas in our review we observed beneficial effects of polyphenol supplementation for up to $96 \mathrm{~h}$ post-exercise. While we found beneficial effects on DOMS at 24, 48 and $72 \mathrm{~h}$ post-exercise, Doma et al. [17] only reported positive effects at $24 \mathrm{~h}$ and did not examine responses at the $72 \mathrm{~h}$ timepoint. In a number of studies included in our meta-analysis DOMS was still elevated at $72 \mathrm{~h}$.

Doma et al. [17] found a reduction in markers of muscle damage, inflammation, and oxidative stress at 24 and $48 \mathrm{~h}$ post-exercise whereas, we found no effect at those time-points but observed moderate reductions at $72 \mathrm{~h}$ for PC. Possible explanations for the disagreement between our findings and those of Doma et al. [17] include differences in studies selected for the meta-analysis, focusing on polyphenol-rich food, juices and concentrates from fruit and vegetables whilst excluding extracts, and our decision not to pool markers of inflammation and oxidative stress. When studies measured more than one blood marker of muscle damage, inflammation, or oxidative stress, Doma et al. [17] combined these into a single estimate of effect in their meta-analysis. This is problematic because some of the biochemical markers they combined are not directly comparable. For example, for oxidative stress they combined PC, TBARS, uric acid and superoxide dismutase and these are markers of different processes that may not correlate with each other.

Hill et al. [58] conducted a systematic review and meta-analysis on the effect of tart cherry (juices, concentrates, and extracts) and recovery from EIMD. They found moderate beneficial effects on muscular power and recovery of muscular strength, and small beneficial effects on DOMS. However, Hill et al. [58] combined all post-exercise time points into a single summary estimate of effect for each outcome marker. This makes direct comparison with our meta-analysis impossible because we investigated the effects at each post-exercise time point separately in order to capture the time course of recovery and to avoid unit of analysis errors [20,29]. We found weaker evidence than Hill et al. [58] that tart cherry supplementation enhanced recovery after EIMD with statistically significant effects on MIVC, DOMS and CMJ only at a limited number of post-exercise time points (see Supplementary File 9).

\subsection{Limitations}

The results of this systematic review and meta-analysis should be interpreted cautiously considering several limitations in the included studies. Ten of the 25 studies included in this meta-analysis were categorised as high risk of bias but when these were removed in a sensitivity analysis the beneficial effects of polyphenol supplementation were attenuated. There was moderate to substantial heterogeneity for several outcomes across various time points. Many of our subgroup analyses were conducted on a small number of studies which limited the statistical power to detect differences.

The studies included in the meta-analyses varied in design. Of the 25 studies, only five $[38,39,43,45,47]$ recruited well-trained athletes (11.3\% of the total participants). Moreover only $19.4 \%$ of the participants included in this review were female. Male and female participants were combined in five studies [11,35-37,44]. It has been reported that oestrogen may protect muscle from injury by reducing inflammation [59], however, these studies failed to report whether they controlled for stages of the menstrual cycle. Studies varied in the duration outcomes markers were measured post EIMD ranging from immediately to $96 \mathrm{~h}$ after exercise. The studies of shorter duration (i.e., up to $48 \mathrm{~h}$ ) may not have accurately captured the time-course of response for some markers of recovery [2].

Five studies included in this review did not report the polyphenolic composition of the products they administered. Moreover, when polyphenol composition was reported, 
authors mainly relied on published data rather than analysing the batch of polyphenol supplement they used. Because of limited and possibly unreliable data on the polyphenol content of supplements used we could not explore a dose response relationship in this meta-analysis.

We downgraded the quality of the evidence for all primary outcomes across all timepoints to moderate- to very-low. The main reasons for downgrading were serious risk of bias, small overall sample sizes, moderate to substantial heterogeneity and imprecision in summary effect estimates.

\subsection{Future Research}

To clarify the effects of polyphenols on recovery from EIMD there is a need for future studies to accurately characterise the composition of the products used, identify the optimal dose, measure the appearance of polyphenolic metabolites in urine and blood, and utilise tissue markers of muscle damage, inflammation, and antioxidant status. It remains unclear what the optimal duration of polyphenol supplementation is and whether ingestion should start before or after muscle damage has incurred. A possible concern of long-term polyphenol supplementation is that it may blunt important stress signals required for the adaptive response to exercise [3] however, this requires confirmation. This review highlighted that females were underrepresented in the literature and no studies had directly compared the differences in the effects of polyphenol supplementation on recovery between sexes, thus future research opportunities exist to address this gap. The majority of participants included in the studies were recreationally-trained athletes. It is important to confirm the effects of polyphenol supplementation in well-trained and elite athletes who are more likely to experience limited recovery times between competitions. All of the studies included in this review were rated as 'some concerns' or 'high' risk of bias. Thus, there is a need for higher quality randomised controlled trials investigating polyphenols on recovery from EIMD.

\section{Conclusions}

Our systematic review and meta-analysis found moderate to very low certainty evidence that polyphenol-rich foods, juices, and concentrates accelerate recovery of muscle function (up to 13\%) and reduce muscle soreness (up to $29 \%$ ) after EIMD. This magnitude of enhanced recovery could benefit athletes in scenarios where there is limited time between competitive events and impaired recovery could negatively impact performance. Polyphenol supplementation may be useful in situations where EIMD impairs muscle function for extended periods of time such as after very heavy training loads, or at the initiation of a training programme. The mechanisms through which polyphenol supplementation enhances recovery is uncertain, but inhibition of inflammation and enhanced antioxidant capacity may be important. That said, in this review, little evidence of a reduction in either inflammation or oxidative stress was evident. This could reflect limitations in the blood-based methods used by the studies included in this review. Future studies should adopt robust methods of determining inflammation and redox status within muscle tissue to unravel the mechanisms through which polyphenols enhance recovery from EIMD.

In conclusion, our review suggests that consumption of polyphenol-rich foods, juices, and concentrates provides practitioners and athletes with a low risk, food-first approach for enhancing recovery in scenarios where optimising rapid recovery is key. Further investigation is required to determine the optimal duration and dosage of polyphenol supplementation and explore whether enhanced recovery comes at the expense of impaired adaptation. There is also a need for more studies to investigate the effect in well-trained and elite athletes.

Supplementary Materials: The following are available online at https: / www.mdpi.com/article / 10.3390/nu13092988/s1, Supplementary File 1: Database search strategy; Supplementary File 2: Funnel Plots; Supplementary File 3: Quality assessment; Supplementary File 4: Sensitivity analysis; Supplementary File 5: creatine kinase forest plot; Supplementary File 6: C-reactive protein forest 
plot; Supplementary File 7: Interleukin-6 forest plot; Supplementary File 8: Protein carbonyls forest plot; Supplementary File 9: Analysis by individual polyphenol-rich foods; Supplementary File 10: Polyphenol restriction forest plot.

Author Contributions: Conceptualization, M.K.R. and A.L.; methodology, M.K.R., A.L. and L.R.; formulation of search strategy, D.H.; formal analysis, M.K.R., L.R. and A.L.; data curation, M.K.R., L.R. and A.L.; writing-review and editing, M.K.R., A.L., L.R., M.E.B., M.R. and D.H. All authors have read and agreed to the published version of the manuscript.

Funding: This research was funded by Sheffield Hallam University.

Institutional Review Board Statement: This study was approved by the Sheffield Hallam University Ethics Review Process (ER25507017).

Informed Consent Statement: Not applicable.

Data Availability Statement: Data are contained within the article or available from the included studies that have been cited throughout.

Conflicts of Interest: A.L. and M.E.B. have previously received funding from the Cherry Marketing Institute to investigate the effects of cherry on gout. The other authors declare no competing interests.

\section{References}

1. Bowtell, J.; Kelly, V. Fruit-derived polyphenol supplementation for athlete recovery and performance. Sports Med. 2019, 49, 3-23. [CrossRef]

2. Clarkson, P.M.; Hubal, M.J. Exercise-induced muscle damage in humans. Am. J. Phys. Med. Rehabil. 2002, 81, S52-S69. [CrossRef] [PubMed]

3. Owens, D.J.; Twist, C.; Cobley, J.N.; Howatson, G.; Close, G.L. Exercise-induced muscle damage: What is it, what causes it and what are the nutritional solutions? Eur. J. Sport Sci. 2019, 19, 71-85. [CrossRef]

4. Howatson, G.; van Someren, K.A. The prevention and treatment of exercise-induced muscle damage. Sports Med. 2008, 38, 483-503. [CrossRef] [PubMed]

5. Morgan, D.L.; Proske, U. Popping sarcomere hypothesis explains stretch-induced muscle damage. Clin. Exp. Pharm. Physiol. 2004, 31, 541-545. [CrossRef]

6. Fridén, J.; Sjöström, M.; Ekblom, B. Myofibrillar damage following intense eccentric exercise in man. Int. J. Sports Med. 1983, 4, 170-176. [CrossRef]

7. Butterfield, T.A.; Best, T.M.; Merrick, M.A. The dual roles of neutrophils and macrophages in inflammation: A critical balance between tissue damage and repair. J. Athl. Train. 2006, 41, 457-465.

8. Williamson, G. The role of polyphenols in modern nutrition. Nutr. Bull. 2017, 42, 226-235. [CrossRef] [PubMed]

9. Connolly, D.A.; McHugh, M.P.; Padilla-Zakour, O.I.; Carlson, L.; Sayers, S.P. Efficacy of a tart cherry juice blend in preventing the symptoms of muscle damage. Br. J. Sports Med. 2006, 40, 679-683; discussion 683. [CrossRef] [PubMed]

10. Trombold, J.R.; Reinfeld, A.S.; Casler, J.R.; Coyle, E.F. The effect of pomegranate juice supplementation on strength and soreness after eccentric exercise. J. Strength Cond. Res. 2011, 25, 1782-1788. [CrossRef]

11. Lynn, A.; Garner, S.; Nelson, N.; Simper, T.N.; Hall, A.C.; Ranchordas, M.K. Effect of bilberry juice on indices of muscle damage and inflammation in runners completing a half-marathon: A randomised, placebo-controlled trial. J. Int. Soc. Sports Nutr. 2018, 15, 1-8. [CrossRef]

12. McLeay, Y.; Barnes, M.J.; Mundel, T.; Hurst, S.M.; Hurst, R.D.; Stannard, S.R. Effect of New Zealand blueberry consumption on recovery from eccentric exercise-induced muscle damage. J. Int. Soc. Sports Nutr. 2012, 9, 19. [CrossRef] [PubMed]

13. Clifford, T.; Berntzen, B.; Davison, G.W.; West, D.J.; Howatson, G.; Stevenson, E.J. Effects of Beetroot Juice on Recovery of Muscle Function and Performance between Bouts of Repeated Sprint Exercise. Nutrients 2016, 8, 506. [CrossRef] [PubMed]

14. Morgan, P.T.; Wollman, P.M.; Jackman, S.R.; Bowtell, J.L. Flavanol-Rich Cacao Mucilage Juice Enhances Recovery of Power but Not Strength from Intensive Exercise in Healthy, Young Men. Sports 2018, 6, 159. [CrossRef] [PubMed]

15. Bahadoran, Z.; Mirmiran, P.; Jeddi, S.; Azizi, F.; Ghasemi, A.; Hadaegh, F. Nitrate and nitrite content of vegetables, fruits, grains, legumes, dairy products, meats and processed meats. J. Food Compos. Anal. 2016, 51, 93-105. [CrossRef]

16. Kujala, T.S.; Vienola, M.S.; Klika, K.D.; Loponen, J.M.; Pihlaja, K. Betalain and phenolic compositions of four beetroot (Beta vulgaris) cultivars. Eur. Food Res. Technol. 2002, 214, 505-510. [CrossRef]

17. Doma, K.; Gahreman, D.; Connor, J. Fruit supplementation reduces indices of exercise-induced muscle damage: A systematic review and meta-analysis. Eur. J. Sport Sci. 2021, 24, 562-579. [CrossRef]

18. Warren, G.L.; Lowe, D.A.; Armstrong, R.B. Measurement tools used in the study of eccentric contraction-induced injury. Sports Med. 1999, 27, 43-59. [CrossRef]

19. Page, M.J.; McKenzie, J.E.; Bossuyt, P.M. The PRISMA 2020 statement: An updated guideline for reporting systematic reviews. BMJ 2021, 372, n71. [CrossRef]

20. Higgins, J.P.T.; Thomas, J.; Chandler, J.; Cumpston, M.; Li, T.; Page, M.J.; Welch, V.A. Cochrane Handbook for Systematic Reviews of Interventions Version; Cochrane: London, UK, 2021. 
21. Starbuck, C.; Eston, R.G. Exercise-induced muscle damage and the repeated bout effect: Evidence for cross transfer. Eur. J. Appl. Physiol. 2012, 112, 1005-1013. [CrossRef]

22. RevMan 5.3 User Guide. Available online: https:/ / training.cochrane.org/sites/training.cochrane.org/files/public/uploads/ resources/downloadable_resources/English/RevMan_5.3_User_Guide.pdf (accessed on 24 June 2014).

23. Sterne, J.A.C.; Savovic, J.; Page, M.J. RoB 2: A revised tool for assessing risk of bias in randomised trials. BMJ 2019, 366, 14898. [CrossRef] [PubMed]

24. Higgins, J.P.T.; Altman, D.; Curtin, F.; Li, T.; Senn, S. Revised Cochrane Risk of Bias Tool for Randomized Trials (RoB 2.0) Additional Considerations for Cross-Over Trials 2016. 2021. Available online: https://www.unisa.edu.au/contentassets/72bf756 06a2b4abcaf7f17404af374ad/rob2-0_indiv_main_guidance.pdf (accessed on 20 October 2016).

25. Machin, D.R.; Christmas, K.M.; Chou, T.; Hill, S.C.; Van Pelt, D.W.; Trombold, J.R.; Coyle, E.F. Effects of differing dosages of pomegranate juice supplementation after eccentric exercise. Physiol. J. 2014, 2014. [CrossRef]

26. Peschek, K.; Pritchett, R.; Bergman, E.; Pritchett, K. The effects of acute post exercise consumption of two cocoa-based beverages with varying flavanol content on indices of muscle recovery following downhill treadmill running. Nutrients 2014, 6, 50-62. [CrossRef]

27. Clifford, T.; Bell, O.; West, D.J.; Howatson, G.; Stevenson, E.J. The effects of beetroot juice supplementation on indices of muscle damage following eccentric exercise. Eur. J. Appl. Physiol. 2016, 116, 353-362. [CrossRef] [PubMed]

28. Lamb, K.L.; Ranchordas, M.K.; Johnson, E.; Denning, J.; Downing, F.; Lynn, A. No effect of tart cherry juice or pomegranate juice on recovery from exercise-induced muscle damage in non-resistance trained men. Nutrients 2019, 11, 1593. [CrossRef]

29. Taylor, K.S.; Mahtani, K.R.; Aronson, J.K. Combining and converting groups when extracting data for meta-analysis. BMJ Evid. Based Med. 2021, 26, 85-87. [CrossRef]

30. Cohen, J. Statistical Power Analysis for the Behavioral Sciences; Academic Press: New York, NY, USA, 1988.

31. Guyatt, G.H.; Thorlund, K.; Oxman, A.D. GRADE guidelines: 13. Preparing summary of findings tables and evidence profilesContinuous outcomes. J. Clin. Epidemiol. 2013, 66, 173-183. [CrossRef]

32. Cumming, G. Understanding the New Statistics: Effect Sizes, Confidence Intervals and Meta-Analyses; Routledge: East Sussex, UK, 2012.

33. Guyatt, G.; Oxman, A.D.; Akl, E.A.; Kunz, R.; Vist, G.; Brozek, J.; Norris, S.; Falck-Ytter, Y.; Glasziou, P.; DeBeer, H. GRADE guidelines: 1. Introduction-GRADE evidence profiles and summary of findings tables. J. Clin. Epidemiol. 2011, 64, 383-394. [CrossRef]

34. Bowtell, J.L.; Sumners, D.P.; Dyer, A.; Fox, P.; Mileva, K.N. Montmorency cherry juice reduces muscle damage caused by intensive strength exercise. Med. Sci. Sports Exerc. 2011, 43, 1544-1551. [CrossRef]

35. Clifford, T.; Allerton, D.M.; Brown, M.A.; Harper, L.; Horsburgh, S.; Keane, K.M.; Stevenson, E.J.; Howatson, G. Minimal muscle damage after a marathon and no influence of beetroot juice on inflammation and recovery. Appl. Physiol. Nutr. Metab. 2017, 42, 263-270. [CrossRef]

36. Howatson, G.; McHugh, M.; Hill, J.; Brouner, J.; Jewell, A.; Van Someren, K.A.; Shave, R.; Howatson, S. Influence of tart cherry juice on indices of recovery following marathon running. Scand. J. Med. Sci. Sports 2010, 20, 843-852. [CrossRef]

37. Kuehl, K.S.; Perrier, E.T.; Elliot, D.L.; Chesnutt, J.C. Efficacy of tart cherry juice in reducing muscle pain during running: A randomized controlled trial. J. Int. Soc. Sports Nutr. 2010, 7, 17. [CrossRef] [PubMed]

38. Abbott, W.; Brashill, C.; Brett, A.; Clifford, T. Tart cherry juice: No effect on muscle function loss or muscle soreness in professional soccer players after a match. Int. J. Sports Physiol. Perform. 2020, 15, 249-254. [CrossRef]

39. Bell, P.G.; Walshe, I.H.; Davison, G.W.; Stevenson, E.J.; Howatson, G. Recovery facilitation with Montmorency cherries following high-intensity, metabolically challenging exercise. Appl. Physiol. Nutr. Metab. 2015, 40, 414-423. [CrossRef] [PubMed]

40. Bell, P.G.; Stevenson, E.; Davison, G.W.; Howatson, G. The effects of Montmorency tart cherry concentrate supplementation on recovery following prolonged, intermittent exercise. Nutrients 2016, 8, 441. [CrossRef] [PubMed]

41. Brown, M.A.; Stevenson, E.J.; Howatson, G. Montmorency tart cherry (Prunus cerasus L.) supplementation accelerates recovery from exercise-induced muscle damage in females. Eur. J. Sport Sci. 2019, 19, 95-102. [CrossRef]

42. Clifford, T.; Howatson, G.; West, D.J.; Stevenson, E.J. Beetroot juice is more beneficial than sodium nitrate for attenuating muscle pain after strenuous eccentric-bias exercise. Appl. Physiol. Nutr. Metab. 2017, 42, 1185-1191. [CrossRef]

43. Daab, W.; Bouzid, M.A.; Lajri, M.; Bouchiba, M.; Saafi, M.A.; Rebai, H. Chronic beetroot juice supplementation accelerates recovery kinetics following simulated match play in soccer players. J. Am. Coll. Nutr. 2021, 40, 61-69. [CrossRef]

44. Hutchison, A.T.; Flieller, E.B.; Dillon, K.J.; Leverett, B.D. Black currant nectar reduces muscle damage and inflammation following a bout of high-intensity eccentric contractions. J. Diet. Suppl. 2016, 13, 1-15. [CrossRef]

45. Kupusarevic, J.; McShane, K.; Clifford, T. Cherry gel supplementation does not attenuate subjective muscle soreness or alter wellbeing following a match in a team of professional rugby union players: A pilot study. Sports 2019, 7, 84. [CrossRef]

46. Lima, L.C.; Barreto, R.V.; Bassan, N.M.; Greco, C.C.; Denadai, B.S. Consumption of an anthocyanin-rich antioxidant juice accelerates recovery of running economy and indirect markers of exercise-induced muscle damage following downhill running. Nutrients 2019, 11, 2274. [CrossRef] [PubMed]

47. Morehen, J.C.; Clarke, J.; Batsford, J.; Barrow, S.; Brown, A.D.; Stewart, C.E.; Morton, J.P.; Close, G.L. Montmorency tart cherry juice does not reduce markers of muscle soreness, function and inflammation following professional male rugby League match-play. Eur. J. Sport Sci. 2021, 21, 1003-1012. [CrossRef] [PubMed]

48. Quinlan, R.; Hill, J.A. The efficacy of tart cherry juice in aiding recovery after intermittent exercise. Int. J. Sports Physiol. Perform. 2020, 15, 368-374. [CrossRef] 
49. Tashjian, R.Z.; Deloach, J.; Porucznik, C.A.; Powell, A.P. Minimal clinically important differences (MCID) and patient acceptable symptomatic state (PASS) for visual analog scales (VAS) measuring pain in patients treated for rotator cuff disease. J. Shoulder Elb. Surg. 2009, 18, 927-932. [CrossRef]

50. Guyatt, G.H.; Osoba, D.; Wu, A.W. Methods to explain the clinical significance of health status measures. Mayo Clin. Proc. 2002, 77, 371-383. [CrossRef]

51. Manach, C.; Scalbert, A.; Morand, C.; Rémésy, C.; Jiménez, L. Polyphenols: Food sources and bioavailability. Am. J. Clin. Nutr. 2004, 79, 727-747. [CrossRef] [PubMed]

52. Baird, M.F.; Graham, S.M.; Baker, J.S.; Bickerstaff, G.F. Creatine-kinase-and exercise-related muscle damage implications for muscle performance and recovery. J. Nutr. Metab. 2012, 2012. [CrossRef] [PubMed]

53. Brancaccio, P.; Maffulli, N.; Limongelli, F.M. Creatine kinase monitoring in sport medicine. Br. Med. Bull. 2007, 81, 209-230. [CrossRef]

54. Sies, H. Total antioxidant capacity: Appraisal of a concept. J. Nutr. 2007, 137, 1493-1495. [CrossRef]

55. Powers, S.K.; Smuder, A.J.; Kavazis, A.N.; Hudson, M.B. Experimental guidelines for studies designed to investigate the impact of antioxidant supplementation on exercise performance. Int. J. Sport Nutr. Exerc. Metab. 2010, 20, 2-14. [CrossRef]

56. Halliwell, B.; Gutteridge, J.M. Free Radicals in Biology and Medicine; Oxford University Press: New York, NY, USA, 2015.

57. Jajtner, A.R.; Hoffman, J.R.; Townsend, J.R. The effect of polyphenols on cytokine and granulocyte response to resistance exercise. Physiol. Rep. 2016, 4, e13058. [CrossRef] [PubMed]

58. Hill, J.A.; Keane, K.M.; Quinlan, R.; Howatson, G. Tart cherry supplementation and recovery from strenuous exercise: A systematic review and meta-analysis. Int. J. Sport Nutr. Exerc. Metab. 2021, 31, 154-167. [CrossRef] [PubMed]

59. Le, G.; Novotny, S.A.; Mader, T.L.; Greising, S.M.; Chan, S.S.; Kyba, M.; Lowe, D.A.; Warren, G.L. A moderate oestradiol level enhances neutrophil number and activity in muscle after traumatic injury but strength recovery is accelerated. J. Physiol. 2018, 596, 4665-4680. [CrossRef] [PubMed] 